\title{
Planetary transit candidates in CoRoT-LRc01 field ${ }^{\star}$
}

\author{
J. Cabrera ${ }^{1,2}$, M. Fridlund ${ }^{3}$, M. Ollivier ${ }^{4}$, D. Gandolfi ${ }^{5}$, Sz. Csizmadia ${ }^{1}$, R. Alonso ${ }^{6,7}$, S. Aigrain ${ }^{8}$, A. Alapini ${ }^{8}$,
} J.-M. Almenara ${ }^{9}$, P. Barge ${ }^{6}$, A. S. Bonomo ${ }^{6,10}$, P. Bordé ${ }^{4}$, F. Bouchy ${ }^{11}$, H. Bruntt ${ }^{12}$, L. Carone ${ }^{13}$, S. Carpano ${ }^{3}$, H. J. Deeg ${ }^{9}$, R. De la Reza ${ }^{14}$, M. Deleuil ${ }^{6}$, R. Dvorak ${ }^{15}$, A. Erikson ${ }^{1}$, M. Gillon ${ }^{16,7}$, P. Gondoin ${ }^{3}$, E. W. Guenther ${ }^{5}$, T. Guillot ${ }^{17}$, M. Hartmann ${ }^{5}$, A. Hatzes ${ }^{5}$, G. Hebrard ${ }^{11}$, L. Jorda ${ }^{6}$, H. Lammer ${ }^{18}$, A. Léger ${ }^{4}$, A. Llebaria ${ }^{6}$, C. Lovis ${ }^{7}$, P. Magain ${ }^{19}$, M. Mayor ${ }^{7}$, T. Mazeh ${ }^{20}$, C. Moutou ${ }^{6}$, A. Ofir ${ }^{21}$, M. Pätzold ${ }^{13}$, F. Pepe ${ }^{7}$, F. Pont ${ }^{8}$, D. Queloz ${ }^{7}$, M. Rabus ${ }^{9}$, H. Rauer ${ }^{1,22}$, C. Régulo ${ }^{9,23}$, S. Renner ${ }^{1,24,25}$, D. Rouan $^{12}$, B. Samuel ${ }^{4}$, A. Santerne ${ }^{6}$, J. Schneider ${ }^{2}$, A. Shporer ${ }^{20}$, B. Stecklum ${ }^{5}$, B. Tingley ${ }^{9}$, S. Udry ${ }^{7}$, and G. Wuchterl ${ }^{5}$

1 Institute of Planetary Research, German Aerospace Center, Rutherfordstrasse 2, 12489 Berlin, Germany e-mail: juan.cabrera@dlr.de

${ }^{2}$ LUTH, Observatoire de Paris, CNRS, Université Paris Diderot, 5 place Jules Janssen, 92190 Meudon, France

3 Research and Scientific Support Department, ESTEC/ESA, PO Box 299, 2200 AG Noordwijk, The Netherlands

4 Institut d'Astrophysique Spatiale, Université Paris XI, 91405 Orsay, France

Thüringer Landessternwarte, Sternwarte 5, Tautenburg 5, 07778 Tautenburg, Germany

${ }^{6}$ Laboratoire d'Astrophysique de Marseille, UMR 6110, CNRS/Université de Provence, 38 rue F. Joliot-Curie, 13388 Marseille, France

7 Observatoire de Genève, Université de Genève, 51 chemin des Maillettes, 1290 Sauverny, Switzerland

8 School of Physics, University of Exeter, Stocker Road, Exeter EX4 4QL, UK

9 Instituto de Astrofísica de Canarias, E-38205 La Laguna, Tenerife, Spain

10 INAF - Osservatorio Astrofisico di Catania, via S. Sofia 78, 95123 Catania, Italy

11 Institut d'Astrophysique de Paris, Université Pierre \& Marie Curie, 98bis Bd Arago, 75014 Paris, France

12 LESIA, Observatoire de Paris-Meudon, 5 place Jules Janssen, 92195 Meudon, France

13 Rheinisches Institut für Umweltforschung an der Universität zu Köln, Aachener Strasse 209, 50931, Germany

${ }^{14}$ Observatório Nacional, Rio de Janeiro, RJ, Brazil

15 University of Vienna, Institute of Astronomy, Türkenschanzstr. 17, 1180 Vienna, Austria

${ }^{16}$ Institut d'Astrophysique et de Géophysique, Université de Liège, 17 Allée du 6 Août, Bât. B5C, Liège 1, Belgium

17 Observatoire de la Côte d'Azur, Laboratoire Cassiopée, BP 4229, 06304 Nice Cedex 4, France

18 Space Research Institute, Austrian Academy of Science, Schmiedlstr. 6, 8042 Graz, Austria

19 University of Liège, Allée du 6 août 17, Sart Tilman, Liège 1, Belgium

${ }^{20}$ Wise Observatory, Tel Aviv University, Tel Aviv 69978, Israel

21 School of Physics and Astronomy, Raymond and Beverly Sackler Faculty of Exact Sciences, Tel Aviv University, Tel Aviv, Israel

22 Center for Astronomy and Astrophysics, TU Berlin, Hardenbergstr. 36, 10623 Berlin, Germany

23 Dpto. de Astrofísica, Universidad de La Laguna, 38206 La Laguna, Tenerife, Spain

24 Laboratoire d'Astronomie de Lille, Université de Lille 1, 1 impasse de l'Observatoire, 59000 Lille, France

25 Institut de Mécanique Céleste et de Calcul des Ephémérides, UMR 8028 du CNRS, 77 avenue Denfert-Rochereau, 75014 Paris, France

Received 12 June 2009 / Accepted 27 July 2009

\section{ABSTRACT}

\begin{abstract}
Aims. We present here the list of planetary transit candidates detected in the first long run observed by CoRoT: LRc01, towards the galactic center in the direction of Aquila, which lasted from May to October 2007.

Methods. we analyzed 3719 (33\%) sources in the chromatic bands and 7689 in the monochromatic band. Instrumental noise and the stellar variability were treated with several detrending tools, on which subsequently several transit search algorithms were applied. Results. Forty two sources were classified as planetary transit candidates and up to now 26 cases have been solved. One planet (CoRoT-2b) and one brown-dwarf (CoRoT-3b) have been the subjects of detailed publications.
\end{abstract}

Key words. techniques: photometric - techniques: radial velocities - techniques: spectroscopic - stars: planetary systems binaries: eclipsing

* The CoRoT space mission, launched on December 27 2006, was developed and is operated by CNES, with contributions from Austria, Belgium, Brazil, ESA, Germany and Spain. The first CoRoT data are available to the community from the CoRoT archive:

http://idoc-corot.ias.u-psud. fr.

\section{Introduction}

In the present paper we report the initial results of the third pointing of CoRoT (Baglin et al. 2006) that was carried out in the direction towards the constellation of Aquila (LRc01 coordinates: $\left.19^{\mathrm{h}} 23^{\mathrm{m}} 33.60^{\mathrm{s}} ; 0^{\circ} 27^{\prime} 36^{\prime \prime}\right)$, in the same spirit as the papers on the 
IRa01 by Carpano et al. 2009 and Moutou et al. 2009. We first describe the actual characterization of the field and the methods used in order to detect exoplanetary candidates in the light curves. We then describe the actual follow-up observations carried out and describe the conclusion in each specific case.

In the first selection of candidates in the LRc01 field, 42 candidates were selected for follow-up and characterization. Of these 26 have been settled to date. There are 2 bona fide planets recorded, (Alonso et al. 2008 and Bouchy et al. 2008; Deleuil et al. 2008), the latter of which at 21 Jupiter masses is tentatively classified as a brown dwarf orbiting a solar type star. There are 16 open cases remaining and under further investigation (see below). The remainder of the settled cases are deemed either binaries, grazing binaries or contaminating eclipsing binaries (CEB) where light from an eclipsing binary is blended to the light of a nearby star to produce an event similar to what is expected from a transiting exoplanet in the CoRoT target signal.

\section{Field characterization}

The 11408 targets observed by CoRoT were selected using the information gathered in the database Exo-Dat (Meunier et al. 2007; Deleuil et al. 2009), built with dedicated ground based photometric observations in the visible and near IR bands from 2MASS catalog. CoRoT is a double-purpose mission and the fields observed must achieve a compromise between the requirements of the seismology and the exoplanet science cases. The former requires a small number of bright, well-selected stars and the latter needs a large number of targets due to the relatively low geometric probability (around 5\%) of observing a transit, hence fainter stars are observed. Figures 1 and 2 show color-magnitude diagrams of the stars in LRc01 field. Although there is some degeneracy for low-luminosity objects, we can infer from these diagrams that there is a significant fraction of giant stars, up to 58\% (see the discussion in Aigrain et al. 2009). Giants are not the best candidates for searching for planets because of their large radius (a Jupiter size planet passing in front of a 6 solar radius star produces a drop in the flux of $0.03 \%$ or $0.3 \mathrm{mmag}$ ). This reduces the number of optimal targets for the planetary search, although the giants in the field can still produce interesting science thanks to the considerable capabilities of CoRoT (see for example Michel et al. 2008; Gondoin \& et al. 2009; Hekker et al. 2009; de Ridder et al. 2009). However, the reduced fraction of dwarf stars has to be taken into account when evaluating the performance of the mission (see Sect. 3).

The precision with which planetary parameters can be measured from photometry relies on the precision for the stellar parameters of the host star. Stellar limb darkening parameters in particular are crucial to this analysis. In theory, these can be obtained from pure photometric analysis, but in practice the degeneracy between the inclination and the limb darkening values and the signal-to-noise ratio required to fully characterize the whole set of parameters make it advisable to fix the limb darkening coefficients (see, for example, Torres et al. 2008). However, this requires a knowledge of the stellar nature which cannot be obtained from CoRoT photometry, requiring input from catalogs or complementary ground-based observations. For CoRoT candidates, we make use of the Exo-Dat database, which includes $B, V, R$, and $I$ magnitudes for each target, as well as $J$, $H$, and $K_{\mathrm{s}}$ from the 2MASS survey, from which temperatures and spectral classes, and thereby limb darkening coefficients, can be estimated.

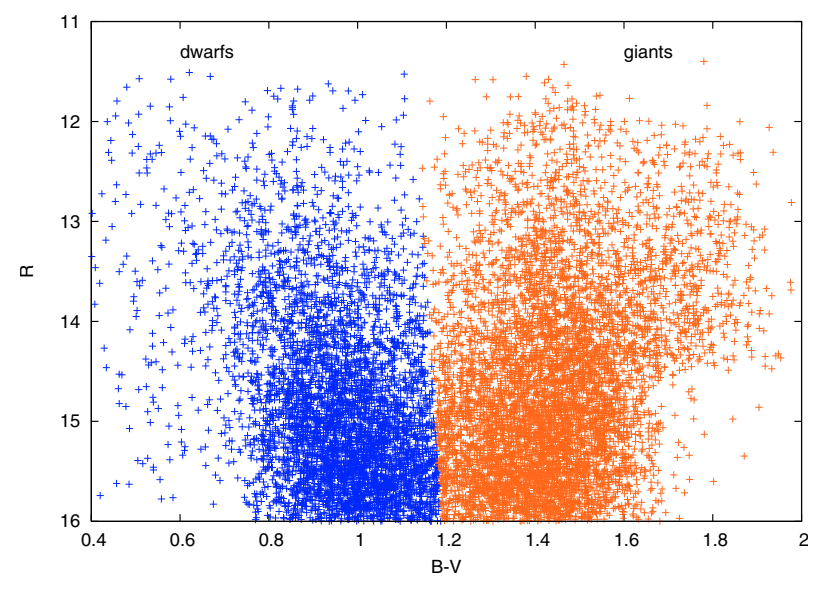

Fig. 1. $B-V$ versus $R$ color-magnitude diagram of the stars in LRc01 field. Dwarf stars are most likely to be found in the left part of the diagram, giants in the right part.

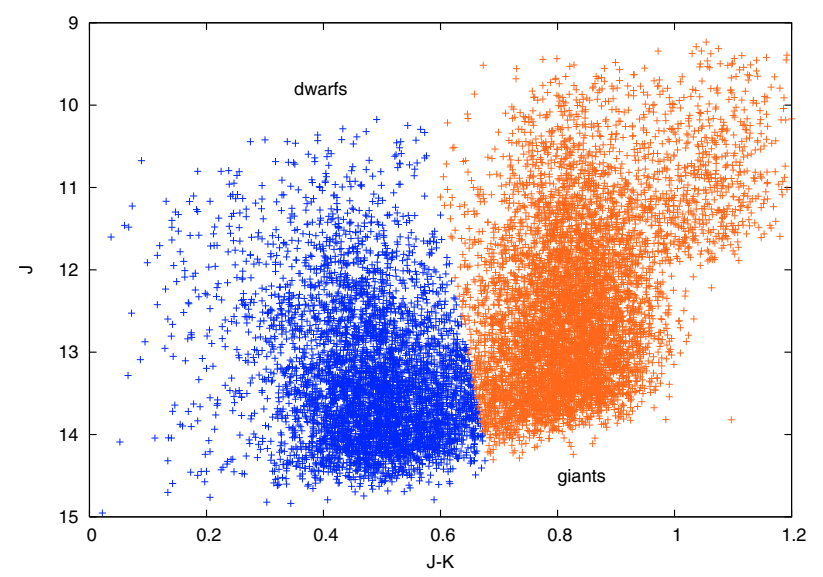

Fig. 2. $J-K$ versus $J$ color-magnitude diagram of the stars in LRc01 field for comparison with Fig. 1. The axes of the figure are fixed for convenience; however, this means that star 101363195, a white dwarf candidate with a $J-K$ of -0.45 and variable with a period of 0.98 days, is not shown.

\section{Data reduction}

CoRoT samples the stars with a cadence of 512 s (see Surace et al. 2008). However, for a limited number of candidates, a higher cadence of $32 \mathrm{~s}$ is available. A bi-prism was installed to disperse the light in the Exoplanet CCD, which helps to distinguish achromatic planetary transits and chromatic stellar variability. For bright stars (32.6\% of the targets in the LRc01 field), the flux is measured in three separate channels: red, green, and blue; which have no direct correspondence to any standard photometric band. For the rest of the targets, the flux is measured in a single white channel. For a complete description of the observing modes of the satellite, please refer to Boisnard \& Auvergne (2006); Barge et al. (2008b), and Auvergne et al. (2009).

Data is furnished to the detection teams at the so-called N2 level (Baudin et al. 2006), the same data made public one year later through the CoRoT archive: http://idoc-corot.ias. u-psud.fr. Several treatments have already been applied to eliminate some of the main systematic sources of error (see Drummond et al. 2008; Auvergne et al. 2009). Invalid data points, such as the measurements taken during the passage of the satellite through the South Atlantic Anomaly (SAA) are flagged, this systematic source of noise can easily be avoided. However, 


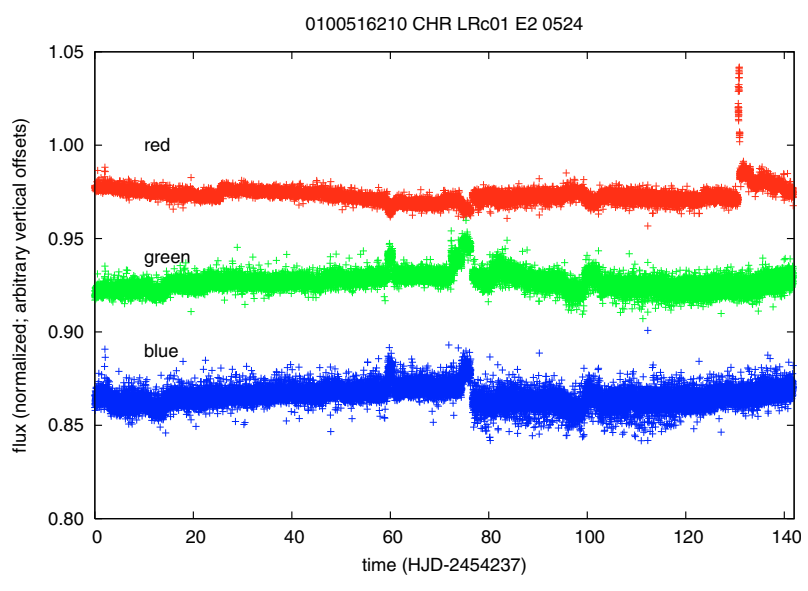

Fig. 3. Example of light curve affected by several instrumental effects. See text for details.

other sources of systematic noise remain, such as hot pixels and, in the version 1.2 of the N2 data released in February 2008 (and the one which is used for the results presented in this paper), effects related to the passage of the satellite through the cone of shadow of the Earth illuminated by the Sun. Each detection team uses different techniques to deal with these problems and it is beyond the scope of this work to describe them. However, we show how colored channels are an useful tool for the filtering.

The nature of hot pixels and their incidence in CoRoT's photometry are described in detail in the following papers: Drummond et al. (2008); Pinheiro da Silva et al. (2008), and Auvergne et al. (2009). In Fig. $3^{1}$ we show a typical light curve affected by several instrumental effects that are not filtered by the pipeline. As hot pixels affect only one pixel at a time, it is easy to tell when one of these events occurs by comparing the evolution of the flux in the different channels. Figure 4 shows a detail from one of these events: a moderate intensity hot pixel appears on the red channel and changes the average value of the measured flux, but it does not affect the other channels. Figure 5 shows a detail with a more complicated pattern: in $T=71$ a small-amplitude hot pixel appears in the red channel; later, in $T=72.5$ a bigger hot pixel affects the green channel, producing a perturbation that lasts over one day before it is relaxed (although with a different value of the average flux measured); in $T=74.5$ there is a change in the flux measured by each channel, much like a redistribution of the flux within the mask, because the total flux remains constant. After a gap in the data in $T=76.5$, the flux redistribution reaches a metastable state that lasts several days. Figure 6 shows the effect of a quite energetic hot pixel in the red channel.

The passage of the satellite through the Earth's umbra is another important source of systematic noise present in version 1.2 of the pipeline (see Auvergne et al. 2009). In future versions of the pipeline, this effect will be flagged and thus be easily avoidable. Nevertheless, the filtering of this phenomenon is relatively straightforward. It is a systematic effect that is present in all light curves, although it has a more severe effect on bright lights curves measured with a small mask (for a description of CoRoT masks, please refer to Llebaria \& Guterman 2006) because of border effects (due to jitter, the flux might fall temporally out of the mask). Figure 7 shows a light curve that is strongly affected by this effect, and Fig. 8 shows a detail of the perturbation. The pattern is relatively stable, and the affected

\footnotetext{
1 A linear regression was subtracted from the flux in each channel when doing the normalization.
}

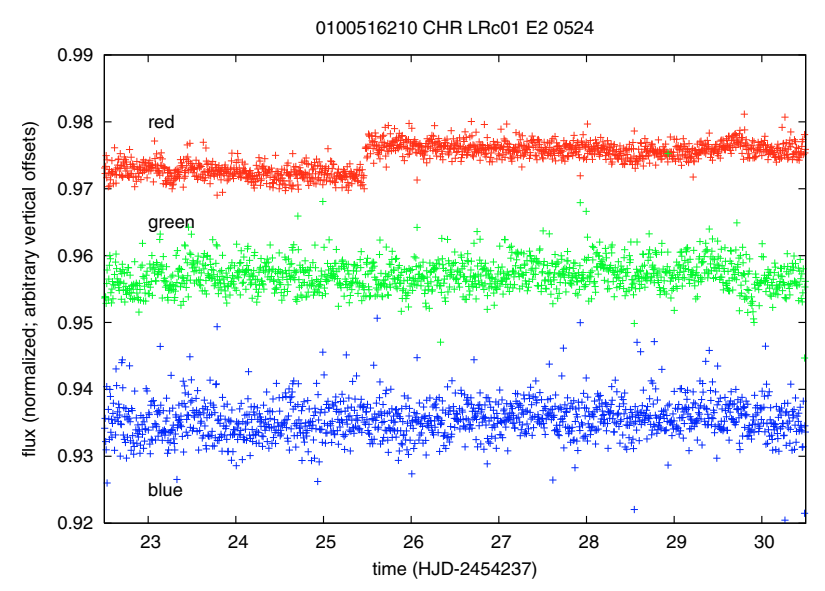

Fig. 4. Detail of the light curve where the red channel is affected by a low-amplitude hot pixel.

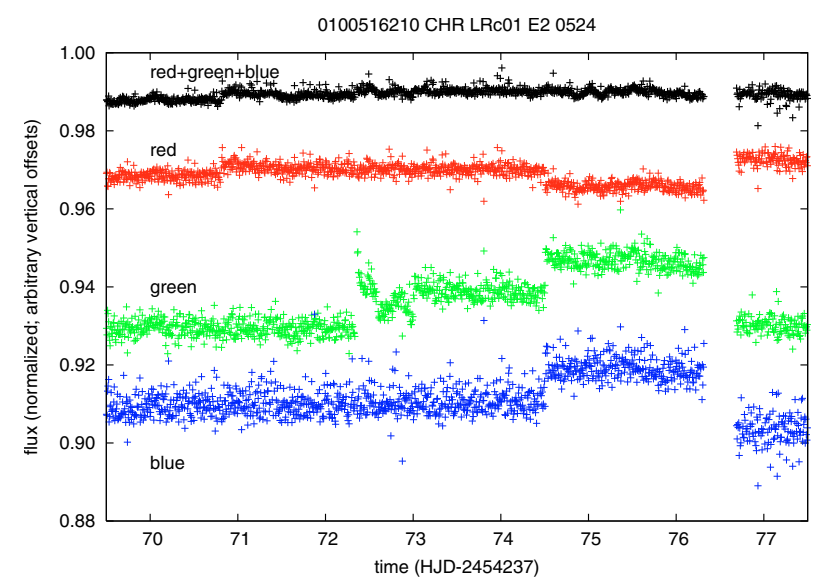

Fig. 5. Detail of the light curve showing different instrumental effects. In $T=71$ a small-amplitude hot pixel appears in the red channel; in $T=72.5$ a bigger hot pixel affects the green channel; in $T=74.5$ there is a change in the flux measured by each channel, but the total flux within the mask remains constant. After the gap in the data in $T=76.5$ the flux distribution remains stable.

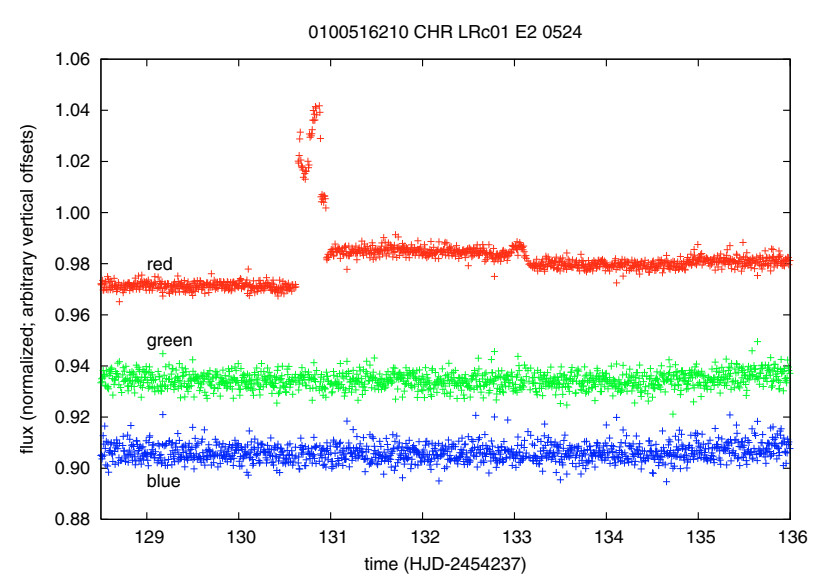

Fig. 6. Detail of the light curve where the red channel is affected by a strong hot pixel, with the change in the relaxation rate around $T=133$.

points can either be treated as outliers or, as the effect appears always at the same time in the reference frame of the satellite, compiled into a list of affected points that can then be discarded. Recently, Mazeh et al. (2009) have published a paper on the filtering of other systematic effects present in the LRa01 run. 


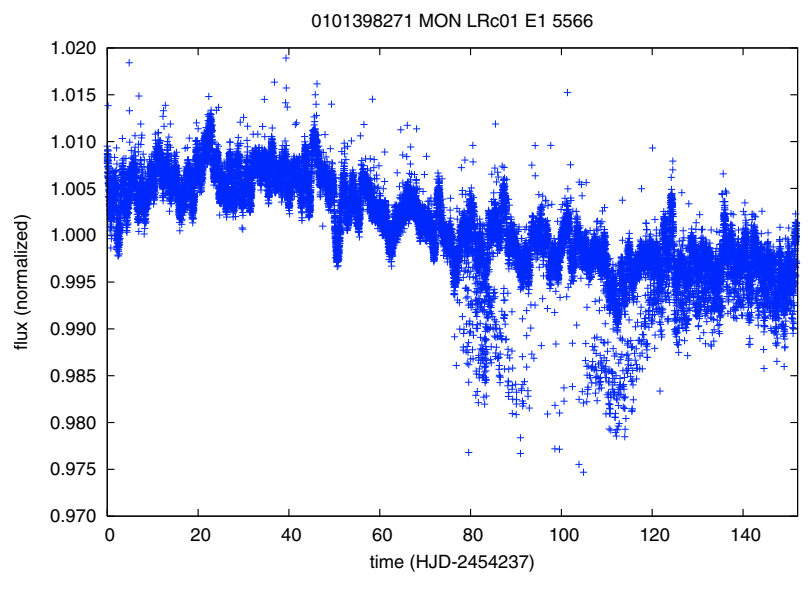

Fig. 7. Light curve affected by systematic sources of noise related to the passage of the satellite through the cone of shadow of the Earth illuminated by the Sun.

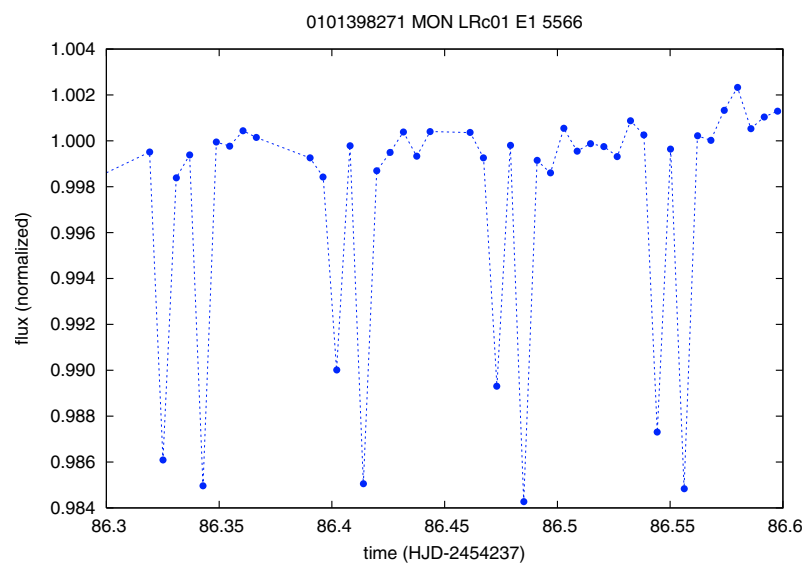

Fig. 8. Detail of the systematic noise related to the passage of the satellite through the Earth's umbra.

This approach appears to reduce the systematic noise in the light curves significantly; although it has not been used in this paper.

The second part of the data processing is the filtering of the stellar activity and the search for transits. Again, each detection team is responsible for constructing and applying its own algorithms. Some of this methods have already been published (Alapini \& Aigrain 2008; Bordé et al. 2007; Carpano \& Fridlund 2008; Moutou et al. 2005, 2007; Régulo et al. 2007; Renner et al. 2008) so do not need to be described. We do, however, discuss their performances below (also treated in Barge 2009 and Auvergne et al. 2009).

\section{Light curve modeling of selected candidates}

For the detailed modeling of the transits of planetary candidates, an IDL $^{2}$ code with a graphical interface was built ${ }^{3}$. It is based on the transit model by Mandel \& Agol (2002) and applies the Amoeba optimization procedure (Press et al. 2002). The code is applied on the filtered and normalized light curves.

\footnotetext{
2 IDL is a registered trademark of ITT corporation.

3 The code can be provided on request to its author: szilard.csizmadia@dlr.de
}

Table 1. List of the detection teams (institutes and people).

\begin{tabular}{cl}
\hline \hline team & participants \\
\hline DLR & Heike Rauer, Stefan Renner, Anders Erikson \\
ESTEC & Malcolm Fridlund, Stefania Carpano \\
U. of Exeter & Suzanne Aigrain, Frédéric Pont, Aude Alapini \\
IAC & Hans Deeg, José Manuel Almenara, Clara Régulo \\
IAS & Pascal Bordé, Benjamin Samuel \\
U. of Köln & Martin Paetzold, Ludmila Carone \\
LAM & Pierre Barge, Roi Alonso \\
LUTh & Jean Schneider, Juan Cabrera \\
\hline
\end{tabular}

For each light curve, we corrected for the impact of possible contaminant sources (the so-called third light) through the following equation:

$$
l\left(\varphi_{i}\right)=\frac{1-\Delta €\left(\varphi_{i}\right)+L_{3}}{1+L_{3}}
$$

where $l\left(\varphi_{i}\right)$ is the observed, normalized light at phase $\varphi_{i}, \Delta \mathrm{Ł}\left(\varphi_{i}\right)$ the light loss of the central star due to the transit of a dark object at the same phase expressed in fractions of the unobscured light of the star, and $L_{3}=\frac{c}{1-c}$ is the contribution of the contaminant source. The denominator normalizes the light curve at the out-of-eclipse part. Variable $c$ is the contamination factor: the fraction of the total observed light that comes from other, unresolved object(s). We assume that it is constant during the observational interval. This contamination factor - only for the unresolved sources - was obtained from the Exo-Dat database ${ }^{4}$.

The parameters that determine the light curve shape are the orbital eccentricity $e$, the argument of the periastron $\omega$, the epoch and period, two limb-darkening coefficients $u_{1}$ and $u_{2}-$ we applied the quadratic limb-darkening law, see Claret $(1998,2000)-$ the planet to stellar radius ratio $R_{\mathrm{p}} / R_{\mathrm{s}}$, the semi-major axis to stellar radius ratio $a / R_{\mathrm{S}}$ and the inclination $i$. We assumed that the orbits are circular, $e=\omega=0$ was set in every case. The limb-darkening coefficients were determined by a bilinear interpolation of the tables in Claret (2000) in the $\log g-T_{\text {eff }}$ plane (we assumed a solar metallicity for the stars for the sake of simplicity), and the parameters of the central star were taken from the Exo-Dat database. We fixed the epoch and period at their values determined from the light curve itself and calculated the phases. To correct for possible small errors in the phase determination, we introduced a phase-shift similar to the one in the WD-code (Wilson \& Devinney 1971), which translates into a constant added to each phase value (found to be very close to zero in every case). Finally, we only had four free parameters: phase-shift, $i, R_{\mathrm{P}} / R_{\mathrm{s}}$, and $a / R_{\mathrm{s}}$. We tried several different starting points and each the four parameters were fitted simultaneously by the Amoeba-procedure mentioned above.

The results of the light curve modeling of the candidate E2_1145 is reported in Sect. 6.3.1.

\section{Results}

Detection teams, shown in Table 1, were assigned the task of detrending and analyzing the data searching for transits. The data was delivered to the teams on 16 February 2008, one year before it was made public. However, this was not the first time

\footnotetext{
${ }^{4}$ this value is present in the header of the fits file of every light curve provided by the CoRoT archive.
} 
that data from the LRc01 had been analyzed. The alarm mode regularly analyzes CoRoT NO data (raw data from the satellite) during the space observations to decide which targets have to be oversampled (observed with the $32 \mathrm{~s}$ cadence) and to allow an early detection of planetary transits, which can be followed-up immediately. Such is the case of all the planets detected so far by CoRoT.

Each detection team applied different techniques to detrending the light curves and searching for transits (see, for example, Alapini \& Aigrain 2008; Bordé et al. 2007; Carpano \& Fridlund 2008; Moutou et al. 2005, 2007; Régulo et al. 2007; Renner et al. 2008). Then all the candidates found by each team were discussed in regular telephonic teleconferences. Most of them were found by several teams (if not all), but some cases were only found by a single team. A certain number were just false alarms, but sometimes real candidates were discovered that felt just under the detection threshold fixed by the rest of the teams. As pointed out by Moutou et al. (2005), one of the main advantages of having different teams using different techniques is that false alarms depend on the method. Candidates were sorted in a priority list whose hierarchy depended on the quality and the planetary likelihood of the signal: the shape of the transit, the possible existence of out-of-transit variations, the possibility of discarding a secondary eclipse (which will be a clear hint of a binary nature of the candidate), the color of the transit, the depth and the duration of the transit (Seager \& Mallén-Ornelas 2003), and the star's spectral type. Although, before discarding for good any candidate we waited for the spectroscopic confirmation of the stellar nature, as the photometric characterization of Exo-Dat might not be $100 \%$ reliable. The aim is to discard as many as possible of the scenarios mimicking planetary transits from photometry (see Pont et al. 2005). The hierarchy established several categories: 1 (first class candidates that showed no problems); 2 and 3 (candidates that showed some problems in any of the properties needed to assess their planetary nature, although they could not be discarded as binaries just from the photometric analysis of the light curve furnished by CoRoT); and finally some problematic candidates were classified as category 4 , where the analysis on these candidates was pursued, although they were generally not followed-up.

The ordered list of candidates was passed to the follow-up team, which decided its own hierarchy as function of the input from the detection teams, the magnitude of the target, and the disponibility of the observational facilities.

The detection team provided a list with 7 priority 1 candidates. Three of them had been previously reported by the alarm mode (including the planet CoRoT-2b and the brown dwarf CoRoT-3b); 6 of the 7 were solved by the follow-up, characterized as one planet (CoRoT-2b), one brown dwarf (CoRoT-3b), two blends, one contaminating eclipsing binary (CEB), and one SB1; one candidate is still being observed. There were 8 priority 2 candidates: 5 solved by the follow-up: two CEBs, one SB1 and two SB2; 3 candidates are still being observed. Fifteen candidates were classified as priority 3: 6 have been solved: one blend, two CEBs, three SB1, and 9 candidates have not been solved so far. Finally, 13 candidates were classified as priority 4.

Table 4 presents those planetary candidates that have not yet been resolved by follow-up observations. Table 5 shows the candidates whose nature has been established from spectroscopic or photometric ground-based observations. Finally, Table 6 is a list of the 158 eclipsing binaries characterized by the detection teams. The scientific task of the detection teams is to provide a characterized set of planetary transiting candidates; although as a natural by-product they build a list of identified eclipsing binaries which is provided here. Some of the binaries (such as E1_1922, E2_0170, E2_1450, E2_3052, E1_1262, and E2_0169) have already been observed by Karoff et al. (2007) within the Berlin Exoplanet Search Telescope (BEST) survey of variable stars in the CoRoT fields. Among the binaries in the list, the following are clearly eccentric: E1_1273, E1_1352, E1_1422, E1_2978, E1_3418, E1_3671, E1_4583, E1_5097, E1_5173, E2_0258, E2_0667, E2_1661, E2_1954, E2_2257, E2_2631, E2_2871, E2_3956, E2_3995, E2_5245. And some show pulsations (like E2_0844). Obviously, also many variable stars were found, but the task of classifying and characterizing the variables is done by another team (see Debosscher et al. 2009). Among the single eclipse events, there is one curiosity, E2_0379, which shows a primary eclipse 13\% deep; 21.1 days later it shows a secondary ( $0.21 \%$ deep) and nothing else during the next 120 days of observation. The simplest explanation is a long-period eccentric binary. Observations taken with IDS at the Isaac Newton Telescope on the Canary Islands reveal that the spectral type of the main star is B8V.

\section{Detailed follow-up of candidates}

\subsection{Ground-based observations}

The CoRoT observations allow us to detect new classes of transiting exoplanets, viz. very small planets (Léger et al. 2009), brown dwarfs (Deleuil et al. 2008), and objects with very long and short periods. A prerequisite for this is, however, a welldeveloped and organized ground-based follow-up program. The CoRoT point-spread function (PSF) covers about 50 pixels (depending on magnitude and color, see Auvergne et al. 2009) and background objects can contribute to the CoRoT light curve, particularly contaminating eclipsing binaries (CEB). They are relatively easily detected, however, by carrying out ground-based photometry during and out of eclipse, since an object some arcseconds distant from the target star will only contribute a small part to the light curve, and therefore needs to have a largeamplitude eclipse. This photometry goes under the designation ON-OFF photometry, and the major problem is having a suitable telescope ready at the right time for the ON observation. We refer to Deeg et al. (2009) for details.

Grazing binaries, intrinsic stellar pulsations, and stellar activity are other "contaminants" where the CoRoT light curve can mimic a transiting planet, particularly one of small amplitude. Both high spectral resolution spectroscopy and high-precision radial velocity spectroscopy are necessary, the latter also for determining the mass and comparing and refining the orbital parameters derived from the light curve. The contaminant candidates identified through this procedure are SB1, SB2, blends, and CEB's.

In the case of both photometry and spectroscopy, CoRoT requires large resources and use both "normal" observations acquired through the time allocation process, particularly at the European Southern Observatory (ESO), the Observatoire d'Haute Provence (OHP), the Instituto Astrofísica de Canarias (IAC), and McDonald observatory. In this context dedicated time also brought into the CoRoT project through partners and collaborations are utilized, such as the Israeli Wise observatory, the HARPS and EULER guaranteed time of the Observatoire de Geneva, the low-resolution spectrograph of the Türinger Landessternwarte Tautenburg (TLS), and ESAs Optical Ground Station (OGS) facility in Tenerife. 


\subsubsection{False positives}

Contaminating eclipsing binary (CEB). This is the case when a background object that falls within the PSF of CoRoT happens to be an eclipsing binary itself. There are cases where the contaminant was brighter than the target, and without knowing spectral types, we do not really know (or care) if contaminants are foreground or background. It will then affect the CoRoT light curve, contributing some fraction of a percent to the light curve and thus be taken for a variation in the target star itself. CEBs can be identified if the CoRoT light curve happens to be a chromatic one and the variation with color is wrong for the target, as was the case in the first identified low-amplitude eclipse signal found in one of the first observations reduced. The variation in the white light curve was just 0.002 , while it was several percent in the blue light curve, thus identifying the variation as belonging to a fainter, eclipsing binary in the blue part of the background. Lacking chromatic light curves, or in complicated cases, ONOFF photometry is carried out as described below.

SB1 (a single-lined spectroscopic binary, where only one component is visible), SB2 (a double-lined spectroscopic binary, where both components are visible), and blends (unresolved triple systems) cannot be solved by photometric observations, because the variability occurs in the target. To confirm the nature of the system, we need to know the mass of the object that is producing the periodic dips in the light curve. SB2 can be easily discarded with a few observations, but other configurations, such as multiple stellar systems, might require more effort.

\subsubsection{Photometry}

The photometry to be carried out can be broadly divided into two classes. First, calibrated color-photometry of potential CoRoT target fields was performed before the CoRoT mission was launched, in order to select the target fine pointings and to characterize the stars in the finally selected fields. This gave rise to the Exo-Cat photometric catalog within the Exo-Dat information system for CoRoT's exoplanetary program. It was built using mainly the Isaac Newton $2.5 \mathrm{~m}$ Telescope (INT) at the Roque de los Muchachos observatory in La Palma, Spain and its WFC camera. This camera has a field of view of 34.5 and a pixel scale of 0.33 . The second instrument used for target selection was the Megacam on the $3.6 \mathrm{~m}$ Canada France Hawaii Telescope (CFHT) on Manua Kea, Hawii. This camera has a $60^{\prime}$ by $60^{\prime}$ field of view with 0 .' 187 pixel resolution.

A photometric follow-up program of individual candidates was initiated to verify that eclipses detected by CoRoT are on the presumed target. It mainly employs ON-OFF photometry, where candidates are observed briefly during a transit, and again offtransit, and their brightness and that of any nearby star is compared. This program and its techniques are described in more detail in Deeg et al. (2009). For the period in question, mainly three instruments have been used. The first is EulerCam on the $1.2 \mathrm{~m}$ Leonard Euler telescope at La Silla, Chile. The second is the Wise observatory in Israel with its $1 \mathrm{~m}$ telescope, using the Princeton Instruments (PI) camera. Finally, the third telescope is the $80 \mathrm{~cm}$ telescope of the Instituto de Astrofísica de Canarias (IAC) at the Observatorio del Teide in Tenerife, Spain. A database of ephemeris is maintained at IAC.

\subsubsection{Spectroscopy}

The spectroscopy is also broadly divided into two classes. First there is the verification of the planet through radial velocity observations. Ideally, a chain of observations are carried out beginning with the identification of SB's using the CORALIE spectrograph on the $1.2 \mathrm{~m}$ Leonard Euler telescope at La Silla and the Sandiford cassegrain echelle spectrograph at McDonald Observatory. After passing this test, the planetary candidate star is observed with SOPHIE, a high-resolution spectrograph on the $1.93 \mathrm{~m}$ telescope at the Observatoire de Haute Provence (OHP), which in high-resolution mode provides a resolution of 76500 over a wavelength range of 3872-6943 A. With various crosscorrelation techniques, this allows radial velocities down to maybe $3-4 \mathrm{~m} / \mathrm{s}$ for the brighter stars in the sample. The final radial velocity stage uses the HARPS spectrograph, which is fiberfed by the $3.6 \mathrm{~m}$ reflector at La Silla Chile. HARPS provides a resolution of 115000 over a wavelength range of 3780-6910 A. For the faint stars in the CoRoT sample, the signal-to-noise in a typical observation is $\sim 20$. Nevertheless, a precision of $2-3 \mathrm{~m} / \mathrm{s}$ can be achieved with a number of well sampled observations, with the best so far for a CoRoT star being $1.2 \mathrm{~m} / \mathrm{s}$ after $\sim 120$ individual observations (Queloz, in preparation).

The second spectroscopic mode is for stellar characterization. For some of the CoRoT candidates, low-resolution spectroscopy $(R \approx 1400-2100)$ was performed using the Nasmyth spectrograph mounted at the 2-m-Alfred Jensch telescope of the Thüringer Landessternwarte Tautenburg (TLS). These observations are useful for assessing the nature of some interesting candidates (Gandolfi et al. 2008), and it eventually triggers further radial velocity follow-ups, as well as identifying and ruling out hot objects (B and early A stars) and giant stars. On the other hand, a high-resolution spectrum is required for determining the stellar parameters, and large telescopes are required, since the majority of the CoRoT targets are faint. For the LRc01 region, essentially all spectra have been obtained using the $8 \mathrm{~m}$ class VLT's of ESO in combination with the UVES and GIRAFFE high-resolution spectrographs - occasionally with the FLAMES multi-fiber positioner as a front-end.

\subsection{Planets}

\subsubsection{LRc01 E1 0523 - CHR - 0101368192}

This object, designated CoRoT-3b, was discovered in the alarm mode after 67 days, whereupon the $512 \mathrm{~s}$ integration time was changed for $32 \mathrm{~s}$ cadence. The follow-up process is described in Deleuil et al. (2008). Originally classed in the Exo-Dat database as a G2V star, the host was found to have a $T_{\text {eff }}$ of $6740 \pm 140 \mathrm{~K}$, after the follow-up spectroscopy carried out with the VLT/UVES combination. With a value of $\log g$ of $\sim 4.2$, it is thus more representative of an early F-type star (F3V, Deleuil et al. 2008). The planetary mass turns out to be $21.66 M_{\text {Jup }}$, and the radius is $1.01 R_{\text {Jup }}$. Deleuil et al. (2008) therefore conclude that this is the first secure inhabitant of the so-called "brown dwarf desert". Maybe more important, the accurately determined radius confirms model predictions of what this parameter is expected to be in an evolved brown dwarf. Burrows et al. (2001) present model calculations, and demonstrate the radius of brown dwarf type objects to be $1 R_{\mathrm{Jup}}$ after $300 \mathrm{Myr}$ and $1 \mathrm{Gyr}$ for intermediate and low-mass objects, respectively. As pointed out by Burgasser etal. (2009), the surface gravity of a $>100$ million year old brown dwarf will be similar to the values derived for this object.

\subsubsection{LRc01 E2 $0192-\mathrm{CHR}-0101206560$}

This object, designated CoRoT-2b (Alonso et al. 2008; Bouchy et al. 2008), was also discovered in alarm mode. The star is 
Table 2. Parameters for the fits of the planetary candidate LRc01_E2_1145 shown in Fig. 9 (see explanation of the parameters in Sect. 4).

\begin{tabular}{rl}
\hline \hline parameter & E2_1145 \\
\hline Period (days; fixed) & $6.212242 \pm 0.000013$ \\
$R_{\mathrm{p}} / R_{\mathrm{s}}$ & $0.0816 \pm 0.0032$ \\
$a / R_{\mathrm{s}}$ & $14.3 \pm 1.4$ \\
$i(\mathrm{deg})$ & $87.1 \pm 0.6$ \\
$e$ (fixed) & 0 \\
$u_{1}$ (fixed) & 0.556 \\
$u_{2}$ (fixed) & 0.212 \\
third light (fixed) & 0.0 \\
\hline
\end{tabular}

visibly highly active with a significant spot activity (Alonso et al. 2008; Lanza et al. 2009). The star is of solar type, with $T_{\text {eff }}$ of $5625 \pm 120 \mathrm{~K}$, a value of $\log g$ of $4.3 \pm 0.2$, a solar metallicity, a radius of $0.92 \pm 0.02 R_{\odot}$, and a $v \sin i$ of about $12 \mathrm{~km} \mathrm{~s}^{-1}$ (Alonso et al. 2008; Bouchy et al. 2008). The high value of $v \sin i$, taken with the value of $i$ derived from the light curve parameters as well as the rotational period derived from the light curve are all consistent with the radius derived from the stellar modeling and all aspects point towards a very young object with an age below 0.5 Gy (Bouchy et al. 2008).

\subsection{Suspected planets or unsettled cases}

This section includes objects that are still being followed-up because their nature as a planetary transit has not been disproved. We also include targets, where there are indications of a nonbinary nature, which has downgraded the priority, and lack of resources have caused them not to be followed-up. The possibility remains of the eclipses being caused by an exoplanetary object, but we stress that these targets are unsettled.

\subsubsection{LRc01 E2 $1145-$ CHR - 0101086161}

Discovered in alarm mode, the follow-up on this candidate has not been finished, but the first radial velocity measurements are compatible with a planetary mass companion to the star, although the possibility of a blend scenario has not been ruled out completely. UVES observations indicate a $T_{\text {eff }}$ of $5350 \mathrm{~K}$, a $\log g$ of 4.4 , and the spectral type of the star is thus K0V. Then assuming a radius of $0.85 R_{\odot}$, the potential planet has a size of $0.7 R_{\text {Jup }}$ (see Table 2 and Fig. 9).

\subsubsection{LRc01 E2 $5801-\mathrm{MON}-0100905839$}

A difficult light curve with many hot pixels and probably some damaged pixels within the readout window. It is a monochromatic light curve, and therefore difficult to filter. Nevertheless, the suspected planetary signal appears clear. The period is relatively long, $15.7 \mathrm{~d}$, the depth of the eclipse is $0.70 \%$, and the eclipse lasts for $3.9 \mathrm{~h}$. The true nature of this candidate has not been definitively established yet because the follow-up is not complete. Anyway, on the basis of the spectral analysis performed on a low S/N UVES spectrum, the object should be a K0 dwarf-star.

We do not present a model of the light-curve as we did with the previous candidate because of the difficulty of assessing the reliability of the fitted parameters. The model of a transiting planet is understood, but the difficulty with this particular light

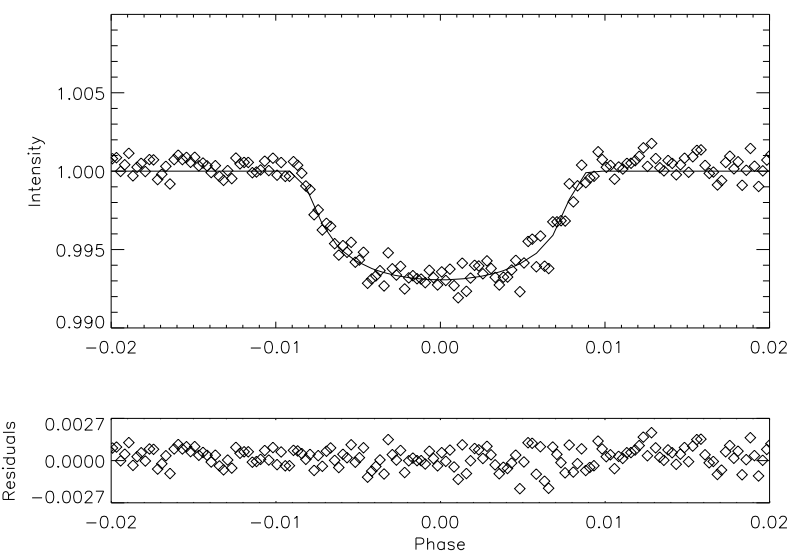

Fig. 9. Normalized, binned, and phase-folded light curve of the candidate LRc01_E2_1145. The transit depth is compatible with a 0.7 Jupiter radii object.

curve is the low signal-to-noise ratio and the impact of the instrumental sources of noise and the hot-pixels. The more aggressive the filtering of those systematics, the more affected the shape of the transit, and it is difficult to calculate how much the parameters obtained from the fit depend on the filtering used. However, we are sure about the periodic signal because we can observe 9 full consecutive transits, and about the size that we infer for the candidate, which is below 1 Jupiter radius if the star is a dwarf and the transit is on target.

\subsubsection{LRc01 E2 $1802-\mathrm{MON}-0100725706$}

This candidate was discovered in alarm mode with a period of $13.239993 \mathrm{~d}$ and a depth of $1.18 \%$. The star was classified as K5V (Exo-Dat), and $m_{V}=15.2$. HARPS observations are not conclusive due to Moon contamination, although the residual variations are compatible with photometric ephemeris and a substellar secondary mass.

\subsubsection{LRc01 E1 1929 - CHR - 0101206989}

This object is classified as $\mathrm{K} 5 \mathrm{~V}$ in the Exo-Dat database and has a $m_{V}=15.0$. The period is only $0.577 \mathrm{~d}$, the depth $0.3 \%$, and the duration $1.3 \mathrm{~h}$. Unfortunately, the signal is only seen in the blue light curve. Radial velocity observations with SOPHIE proved inconclusive (the limit in the variation is as low as $15 \mathrm{~m} / \mathrm{s}$ ); photometry with IAC80 demonstrated that the closest fainter stars that could mimic the transit are not variable. On the basis of lowresolution spectroscopy carried out at TLS, the spectral type of the star is most likely K0III/IV. The follow-up has recently been stopped on this target because some hints of a secondary eclipse had been found in the CoRoT light curve.

\subsubsection{LRc01 E2 $3345-\mathrm{MON}-0101332685$}

This object has a depth of $3.6 \%$, and a period of $13.099 \mathrm{~d}$. Because the star is a K5V of $m_{V}=15.8$, it was observed with HARPS 4 times. The diluted amplitude of the blend is about $60 \mathrm{~m} / \mathrm{s}$, whereas individual error on the measurements range from 22 to $66 \mathrm{~m} / \mathrm{s}$, so the blend variation is hardly significant. 


\subsubsection{LRc01 E1 $4780-M O N-0101186644$}

An $m_{V}=16.0 \mathrm{~K} 0 \mathrm{~V}$ (Exo-Dat) star with a relatively deep $(0.86 \%)$ eclipse with a period of 20.684d. HARPS observations are not conclusive due to Moon contamination. At twice the period determined from the photometry, the radial velocity measurements result in a signal with a few $\mathrm{km} / \mathrm{s}$ amplitude, but this possibility should be confirmed with new observations not affected by Moon contamination.

\subsubsection{LRc01 E1 $0715-\mathrm{CHR}-0101157411$}

This is a single transit that is $9 \mathrm{~h}$ long with a depth of $0.6 \%$. The spectral classification in the Exo-Dat database is G2IV, and its visual magnitude is $m_{V}=13.6$. Two observations with SOPHIE were carried out with a separation of 60 days. No variation was detected and a case of SB1 appears to be excluded. A UVES spectrum was obtained and the following preliminary parameters are derived from this spectrum: the star is roughly solar type, with dwarf characteristics (F8/9 V). It has, however, a high value of $v \sin i, \sim 14 \mathrm{~km} \mathrm{~s}^{-1}$, which makes the interpretation difficult.

\subsubsection{LRc01 E2 4295 - MON - 0101123916}

This candidate has a low priority because it is faint, and there are marginal indications of a secondary in the light curve. The target star has an $m_{V}=16.5$, and a spectral type according to Exo-Dat of M0V. With a period of $1.13828 \mathrm{~d}$ and a depth of the eclipse of $0.28 \%$ (duration $2.9 \mathrm{~h}$ ), it could be a similar case to the CoRoT-7b super Earth (Léger et al. 2009). Photometry is planned for this source in 2009 to discard a CEB scenario.

\subsubsection{LRc01 E1 1632 - CHR - 0101424939}

This source was identified as a binary by two teams and as a low priority planetary candidate by one team. A reanalysis seems to identify a secondary in the light curve, so the object has been placed on hold. The primary is identified as a K0IV from photometry (Exo-Dat) and has a $m_{V}=14.9$.

\subsubsection{LRc01 E2 4390 - MON - 0100768215}

This object has a low priority because two of the detection teams find hints of a secondary eclipse. Nevertheless, photometry carried out at the CFHT and at IAC80 demonstrates that the eclipse is on target. The star is identified as a M0V, with a $m_{V}=16.5$. The period is $2.94 \mathrm{~d}$, the depth $0.3 \%$ and the duration $4.1 \mathrm{~h}$. The nature of this object is still unknown, but its faint magnitude makes it a very difficult target for spectroscopic follow-up observations.

\subsubsection{LRc01 E2 $0934-\mathrm{CHR}-0100887662$}

A single transit of $8.6 \mathrm{~h}$ duration and $2.28 \%$ depth around a G8V star and with $m_{V}=14.1$ from Exo-Dat. It has not been followedup so far.

\subsubsection{LRc01 E1 0673 - CHR - 0100980128}

A candidate with a period of $6.00234 \mathrm{~d}$ and a depth of $0.1 \%$. The Exo-Dat catalog reports a G5IV star with $m_{V}=13.5$. On the basis of a low-resolution spectrum acquired at TLS, we estimated that the candidate might be an F8/G0 dwarf star. Radial velocity follow-up is currently going on for this target.

\subsubsection{LRc01 E1 $2761-\mathrm{MON}-0101439653$}

This is a candidate with a period of $17.49731 \mathrm{~d}$ and a depth of $4.11 \%$ in a K0V (Exo-Dat) star of $m_{V}=15.2$. It has not been followed up.

\subsubsection{LRc01 E1 1791 - CHR - 0101115531}

This is a candidate with low priority because of the shape of the light curve. There is an out of transit variation, and the signal is only found in the red channel, facts which point to a CEB scenario. It has a period of $0.520959 \mathrm{~d}$ and a depth of $0.18 \%$ in a K5V (Exo-Dat) star of $m_{V}=14.9$. It has not been followed up.

\subsubsection{LRc01 E2 $4241-\mathrm{MON}-0100654833$}

This object was only detected by one team and it was kept as priority 3 because the possibility of being a false alarm was not ruled out. Exo-Dat gave K5V and $m_{V}=15.9$. Period is 0.53108d, depth $0.11 \%$, and duration is $2.3 \mathrm{~h}$. Observations with FLAMES indicate an SB1 but is uncertain. Photometry is to be carried out before settling this case definitively.

\subsubsection{LRc01 E1 2278 - MON - 0101614469}

This is a shallow candidate with a long transit, it was flagged as low priority. Exo-Dat gave $\mathrm{K} 5 \mathrm{~V}$ and $m_{V}=15.2$. Low-resolution spectroscopy performed at TLS suggested that the candidate is a G8/K0 giant star. Three HARPS measurements are not conclusive: they are not in phase with CoRoT ephemeris, but individual errors range from 20 to $50 \mathrm{~m} / \mathrm{s}$.

\subsection{Settled cases - non-planetary objects}

\subsubsection{LRc01 E2 5365 - MON - 0100567226}

This candidate shows some variability at the $1 \%$ level, which is not correlated with the transit detection. Two observations with the UVES+FLAMES combination demonstrate radial velocities of $-9.33 \mathrm{~km} \mathrm{~s}^{-1}$ and $-4.56 \mathrm{~km} \mathrm{~s}^{-1}$ at CoRoT phases of 0.62 and 0.87 , respectively. The object is thus classified as type SB1 and further analysis is left for a later time.

\subsubsection{LRc01 E2 3681 - MON - 0110604224}

This candidate is similar to the previous one, a short period (3.45075d) and a slightly long eclipse $(2.8 \mathrm{~h})$ for the period. It is a faint star $m_{V} 15.7$, with a shallow depth of the eclipse $(0,15 \%)$. Although the Exo-Dat database gave G2IV as the spectral type, it was classified in priority 1 . Photometry with EULER during eclipse saw no indication of variation on the target, but a $15 \%$ eclipse on a 1.3 times fainter star, $12^{\prime \prime}$ distant was detected and later confirmed with the IAC80 telescope. This is thus another case of a CEB.

\subsubsection{LRc01 E2 $0305-\mathrm{CHR}-0101351899$}

This is a very interesting target that shows pulsations with a period of 0.168 days. It shows a clear sign of a transit with a period of 10.99 days and depth around $1 \%$. That the light curve is 


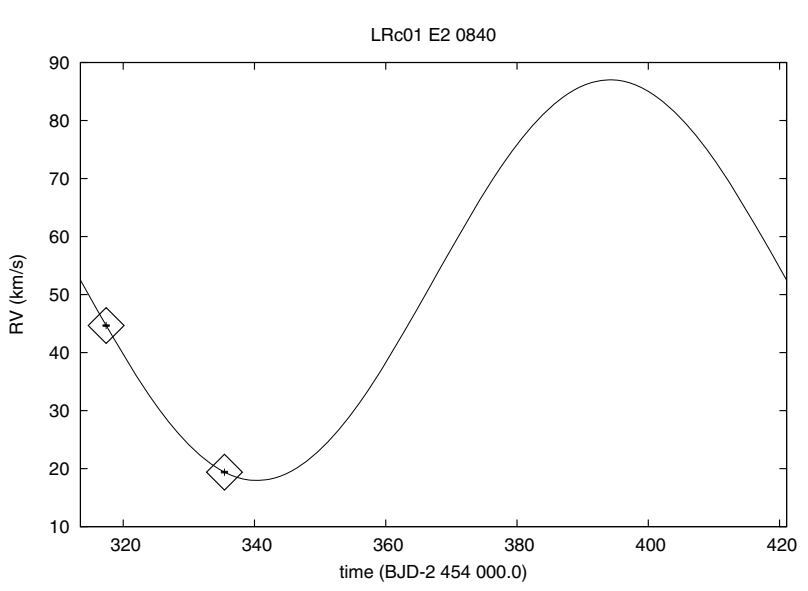

Fig. 10. Radial velocity curve and rough adjustement of an orbital solution with a period of 107.5878 days.

chromatic allowed extensive analysis and it was ranked as a binary by 2 detection teams because of the depth differences between different channels (around 1\% in red and green but $0.5 \%$ in the blue channel). The possibility existed that a background contaminant in the blue channel could account for those depth differences and the candidate finally obtained priority 2 . The Exo-Dat database classified it as G2V and $m_{V}=13$. SOPHIE observations detected a wide single peak, and the second measurement showed a change in radial velocity of $58 \mathrm{~km} \mathrm{~s}^{-1}$, thus, indicating an SB1 type binary, but at twice the period detected by the photometry: 21.97 days.

\subsubsection{LRc01 E1 $3129-\mathrm{MON}-0101436549$}

This is a G2V star (Exo-Dat) of $m_{V}=15.2$, whose light curve looked promising. Low-resolution spectroscopy at TLS indicates a early F star. Photometry with IAC80 showed the eclipse to be on a neighboring, $3.5 \mathrm{mag}$ fainter star $\sim 12^{\prime \prime}$ distant. The eclipse depth is $0.7 \mathrm{mag}$. It is thus a CEB

\subsubsection{LRc01 E1 $0769-$ CHR - 0101068850}

Another single-transit, $7 \mathrm{~h}$ long and 4\% deep on a star classifed as $\mathrm{K} 5 \mathrm{~V}$ by Exo-Dat and with a magnitude of $m_{V}$ 14.0. It was observed twice with SOPHIE and it seems to be an SB2 type binary. The observations are compatible with a period of the binary in the range of 100 to 300 days.

\subsubsection{LRc01 E2 $4006-\mathrm{MON}-0100589010$}

This is another $\mathrm{K} 5 \mathrm{~V}$ star (from Exo-Dat) and a rather faint at $m_{V}=15.9$ one. The period is again quite short $(0.781 \mathrm{~d})$ and it has a depth of $0.14 \%$. Some HARPS observations were carried out, but with no significant result, partly because the spectra had very low signal-to-noise ratio. Finally, photometry at Wise observatory demonstrated that the eclipse was on a background star.

\subsubsection{LRc01 E2 $3895-\mathrm{MON}-0100609705$}

This is a very shallow transit $(0.09 \%)$ with a period of $3.30382 \mathrm{~d}$ on a $m_{V}=15.8 \mathrm{G} 8 \mathrm{IV}$ star (from Exo-Dat). It was immediately identified as a CEB by observations with EULER and IAC80.

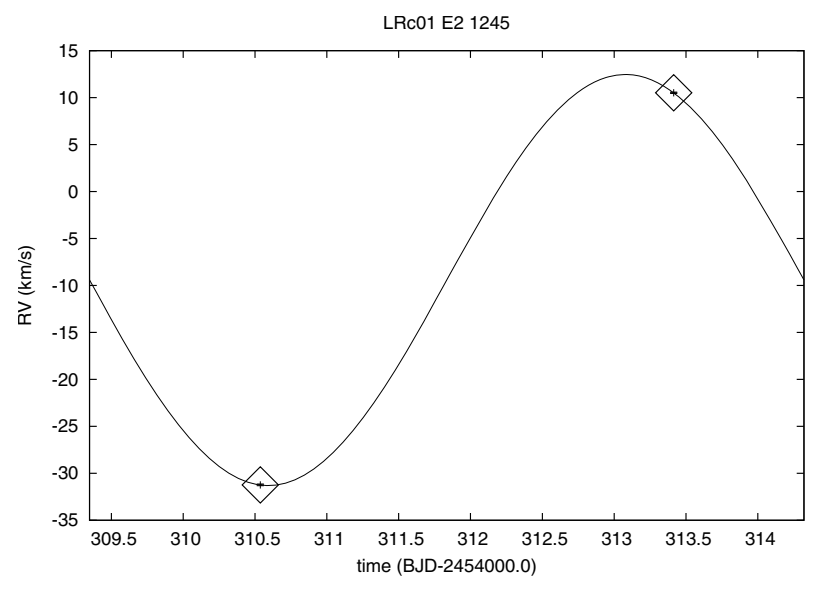

Fig. 11. Radial velocity curve and rough adjustement of an orbital solution with a period of 4.974 days.

\subsubsection{LRc01 E1 $1253-\mathrm{CHR}-0101653417$}

This is a brighter object with a $0.15 \%$ eclipse on a $\mathrm{K} 5 \mathrm{~V}$ $m_{V}=14.6$ star (from Exo-Dat) and with a period of $5.7799 \mathrm{~d}$. Three observations with SOPHIE demonstrated a blend.

\subsubsection{LRc01 E1 1090 - CHR - 0100982006}

This target was discovered in alarm mode, and settled with the first spectra. It is a clear SB1.

\subsubsection{LRc01 E1 2936 - MON - 0101708400}

This is a G2V (Exo-Dat) of $m_{V}=15.2,4.2202209 \mathrm{~d}$ period and a depth of $0.85 \%$. Observations with GIRAFFE+FLAMES indicate an SB1 binary.

\subsubsection{LRc01 E1 2837 - CHR - 0101482707}

This object demonstrates the difficulties with programs that depend on large statistical samples, since the spectral type in ExoDat came out as a G5 super giant of $m_{V}=13.4$. The period was found as $39.8883 \mathrm{~d}$ and the depth of $1.31 \%$ was right for this period if the star was a dwarf. A spectrum was clearly indicated and was obtained by SOPHIE. Unfortunately, the star was shown to be a binary of type SB2.

\subsubsection{LRc01 E2 $0840-\mathrm{CHR}-0101031117$}

This is an interesting case found in alarm mode. The period is $53.8141 \mathrm{~d}$, the depth is $0.60 \%$, and the duration $3.8 \mathrm{~h}$ in a K0V (ExoDaT) of $m_{V}=13.9$. The follow-up classifies it as SB1 but with twice the period formerly proposed by the detection team (see Fig. 10).

\subsubsection{LRc01 E2 1245 - CHR - 0100773735}

This candidate was discovered by alarm mode, and Exo-Dat classifyed it as G8V of $m_{V}=14.0$. The period is $4.974 \mathrm{~d}$, depth $=0.66 \%$, and the duration is somewhat long. From the SOPHIE spectrum it is not possible to clarify whether we have a multiple component binary (SB2 or SB3) or a single component (SB1). In any case, this is a spectroscopic binary and one can clearly measure the movement of one of the components between the extreme phases of the orbit detected by CoRoT (see Fig. 11). 


\subsubsection{LRc01 E1 5626 - MON - 0101259269}

This candidate, $\mathrm{K} 5 \mathrm{~V}$ (Exo-Dat) and $m_{V}=15.9$, was relatively quickly found to have a secondary eclipse and was downgraded to a binary. No follow-up was carried out.

\subsubsection{LRc01 E1 1845 - MON - 0101218359}

This candidate, K5V (Exo-Dat) and $m_{V}=15.2$ was also found to have a secondary eclipse so was downgraded to a binary. No follow-up was carried out.

\subsubsection{LRc01 E2 $2533-\mathrm{MON}-0100468104$}

This candidate, KOIV (Exo-Dat) and $m_{V}=15.2$ was found to have a secondary eclipse so was downgraded to a binary. No follow-up was carried out.

\subsubsection{LRc01 E2 5414 - MON - 0100834293}

This candidate was discovered in alarm mode and with parameters of G0IV and $m_{V}=16.4$ from Exo-Dat. ON-OFF photometry was carried out with the IAC80 and the eclipse was found to be on target. A large secondary at phase 0.41 was in the meantime discovered in the CoRoT light curve (period 11.30d) and it was re-classified as an eccentric binary.

\subsubsection{LRc01 E1 5745 - MON - 0101026464}

This candidate, G2V (Exo-Dat) and $m_{V}=16.3$ was found to have a secondary eclipse and downgraded to a binary. No follow-up was carried out.

\subsubsection{LRc01 E1 3425 - CHR - 0101434308}

This candidate,G2V (Exo-Dat) and $m_{V}=14.8$ was found to have a secondary eclipse and downgraded to a binary. The transit is seen mainly in the blue channel, where it is $2.7 \%$ deep. No follow-up was carried out.

\subsubsection{LRc01 E2 3257 - MON - 0101106246}

Another alarm mode candidate. Spectral type M2V and $m_{V}=16.2$ (from Exo-Dat), with a period of $11.31662 \mathrm{~d}$ and a depth of $0.33 \%$ it appeared promising, but ON-OFF photometry with EULER and IAC80 found a nearby eclipsing binary contaminating the PSF of the target star.

\subsubsection{LRc01 E1 2376 - CHR - 0101095286}

Exo-Dat classified this candidate as K5III and $m_{V}=13.6$. The follow-up demonstrated the binary nature of this object through ON-OFF photometry at IAC80.

\subsubsection{LRc01 E1 4959 - MON - 0101175376}

This case is similar to the previous case (LRc01 E1 2376).

\subsubsection{LRc01 E1 2140 - MON - 0101055792}

This object was found by one of the CoRoT detection teams. Since the eclipse signature was deemed to be slightly too long

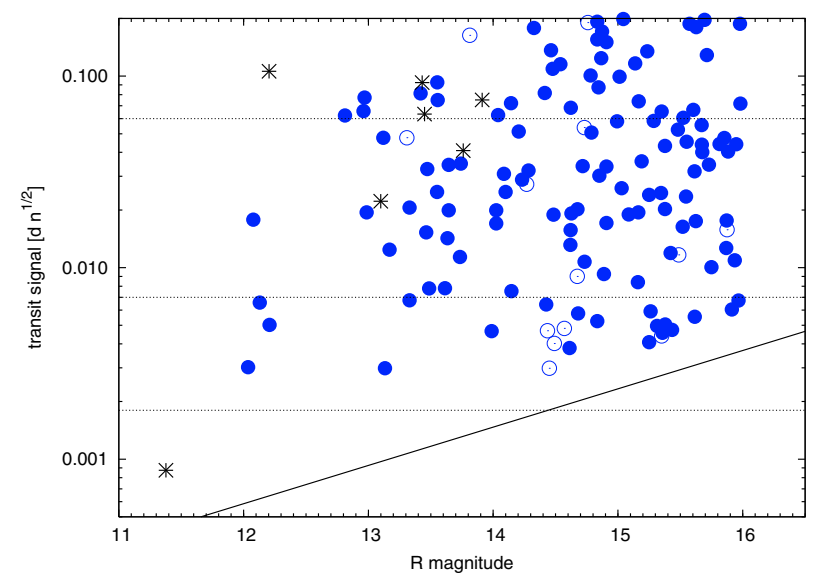

Fig. 12. Transit signal vs. $R$ magnitude for the objects described in this paper. Filled circles are identified objects (Tables 5 and 6) and open circles are for objects in ongoing follow-up (Table 4). The asterisks represent the position of the six planets, and the brown dwarf discovered by CoRoT (CoRoT-7b is in the bottom left corner). The horizontal dashed lines represent (from top to bottom) the expected signal produced by a Jupiter-size planet, a Neptune-size planet, and a 2 Earth-radii planet, respectively.

for the period (i.e. $2.0 \mathrm{~h}$ and $1.9384 \mathrm{~d}$, respectively), low priority was originally assigned to this star; however, the star was classified as a $\mathrm{K} 5 \mathrm{~V}$ in the Exo-Dat database $\left(m_{V}=15.1\right)$. Furthermore, low-resolution spectra taken at TLS indicated a late $\mathrm{G}$ star with a luminosity class compatible with a dwarf or subgiant. On the basis of these results, the radius of the star might be as small as $0.76 R_{\odot}$. The shallow eclipse $(0.13 \%)$ then indicated that the planetary candidate radius could be as small as $2.8 R_{\oplus}$. The target was therefore upgraded to priority 1 in spite of the faint magnitude and transit duration, and further follow-up observations were started. Ground-based observations at IAC80 confirmed that the transit was on target. The object was then observed three times with SOPHIE and scheduled for HARPS follow-up observations when a blend was suspected. Finally, from the HARPS run, the candidate turned out to be a blend with a spatially unresolved eclipsing binary.

\subsubsection{LRc01 E1 $4863-\mathrm{CHR}-0101675703$}

This was a shallow candidate on a $\mathrm{K} 5 \mathrm{~V}$ of $m_{V}=16.0$ (from ExoDat). It was flagged as low priority because one team classified it as a binary since the eclipse was deemed too long $(2.4 \mathrm{~h})$ for the period (1.79308d). HARPS measurements finally concluded that the candidate is a blended eclipsing binary.

\section{Detection statistics}

To measure the performance of a transit survey, it is very useful to follow the approach described by Pont et al. (2006): calculating the noise at the time scales of the transit. This work is described in Aigrain et al. (2009) as it relates to the first three long runs observed by CoRoT. The latter paper conclude that the photometric performance on transit timescales is close to the prelaunch specification, and no significant differences between the three fields are found, apart from a slight gradual degradation of the photometric performance over time, which may be associated with the increase in incidence of hot pixel events. In LRc01, the photometric performance of CoRoT is $7.1 \times 10^{-4}$ at $R=15$ at the sampling of $512 \mathrm{~s}$, conforming to pre-launch specifications. 


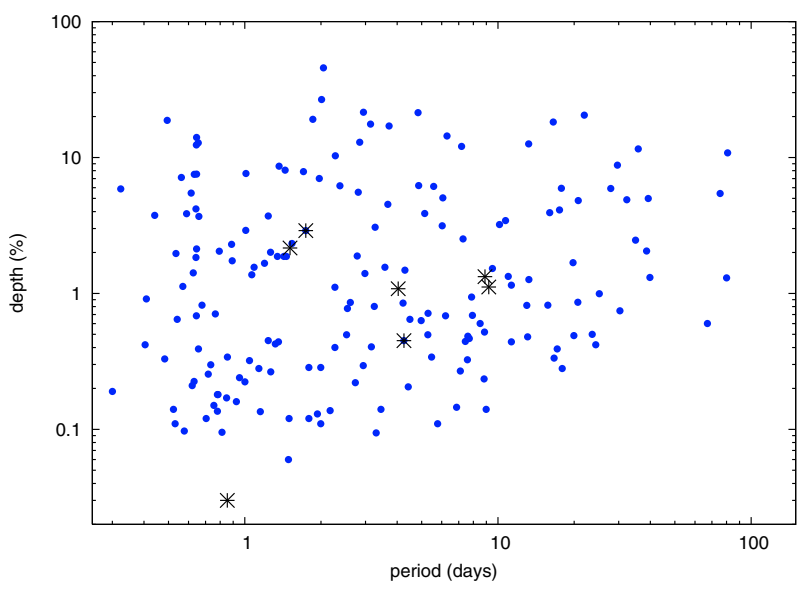

Fig. 13. Depth of the candidates versus their period. The points are for the candidates found in the LRc01 run, and the asterisks are the planetary like objects found by CoRoT. See the interpretation in the text.

Figure 12 represents the transit signal (the product of the relative depth of the transit and the square root of the number of points in the transit) versus the $R$ magnitude. It shows the position of the solved candidates in the LRc01 (Tables 5 and 6) and the ongoing candidates (Table 4), as well as the position of the $6+1$ planetary-like objects discovered by CoRoT ( 6 planets: CoRoT-1b: Barge et al. 2008a; CoRoT-2b: Alonso et al. 2008 and Bouchy et al. 2008; CoRoT-4b: Aigrain et al. 2008 and Moutou et al. 2008; CoRoT-5b: Rauer et al. 2009; CoRoT-6b: Fridlund, in preparation; CoRoT-7b: Léger et al. 2009; and one brown dwarf: CoRoT-3b: Deleuil et al. 2008). In this context, the word "candidate" is used in a broad sense: any target that shows a periodic behavior that can be either a planetary transit or, more likely, an eclipsing binary. The effective detection threshold appears to lie slightly higher than the photon noise limit and a cutoff seems to exist in that no candidate is detected below a transit signal of 0.003 . However, it has to be taken into account that the 0.003 level is only reached for magnitudes brighter than 14 . Only $24 \%$ of LRc01 candidates are in this range of magnitude, and only $7 \%$ in the range brighter than 13 . Probably few of them are dwarf stars. CoRoT-7b clearly stands below the 0.003 threshold, but it was discovered in the LRa01, which had a different stellar population. Probably, the 0.003 threshold (thought to be 0.009 for the IRa01) stems from low number statistics: the short amount of suitable targets in this magnitude range. Taking Fig. 12 into account, the diagrams published by Carpano et al. (2009) and Moutou et al. (2009), as well as the noise studies by Aigrain et al. (2009), it seems plausible to state that CoRoTcan go for Neptune size planets or bigger in all its range of observation (down to $R$ magnitude 16) and for planets as small as 2 Earth radii around stars brighter than $R 14$, although better performances can be achieved around brighter stars (as shown by CoRoT-7b).

However, we can check the performance of the survey in another empirical way, as done by Barge (2009) and Carpano et al. (2009). We can plot, as in Fig. 13, the depth of the candidates found by all teams in the LRc01 against the period of the candidate. In Fig. 13 we have superimposed the candidates in the LRc01 field the depths and periods of the $6+1$ planetary-like objects discovered by CoRoT. A Neptune-like planet moving in front of a solar type star produces a transit of $0.1 \%$, a regime that is well within the reach of CoRoT (for candidates with periods up to 10 days) as pointed out in the previous paragraph.

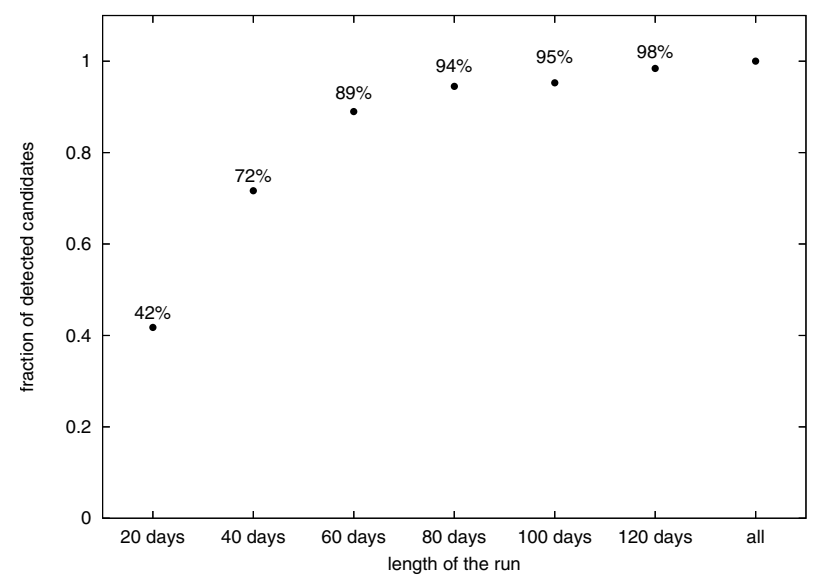

Fig. 14. Evolution of the fraction of candidates detected in a whole run as a function of the length of the run.

However, planets like CoRoT-7b, which is shown in the bottomleft corner of the figure and which has a transit depth of $0.03 \%$, can only be discovered around stars brighter than $R=12$ (following the limits found in Aigrain et al. 2009). There are 317 of such targets in the LRa01(where CoRoT-7b was found), but only 95 in LRc01 (and among those, only 45 are dwarf stars). The targets in this run are fainter and present a larger fraction of giant stars than LRa01 targets (58\% versus 22\%, from Aigrain et al. 2009), which has to be considered when comparing the performances of both runs.

Several papers have been published measuring the performance of the detection algorithms as a function of the length of the run. These analyses were done on simulated data (see, for example, Defaÿ et al. 2001; Carpano \& Fridlund 2008), and the main conclusion is that given a certain number of transits observed, the efficiency of the detection, measured as the smallest radius planet that can be detected around a given star, remains constant when more transits are observed.

In our context, the number of transits observed is given by the length of the run and the period of the target. We can study the efficiency of the detection algorithms as the fraction of candidates found in a shortened run compared to the total number of candidates known in the whole run. Here, a shortened run is built by reducing the length of the light curves; for example taking just the first $20,40,80$, etc. days of observation. The results are given in Fig. 14. For example, analyzing only the first 80 days of the LRc01 we are able to find $94 \%$ of the candidates known in the whole run. Candidates in this context are again both binaries and planets. The detection algorithm finds a list of candidates and a posterior analysis, taking into account not only the significance of the signal, but also the information on the target star and the parameters of the candidate (period, duration, depth, color), in order to distinguish between potential planets and clear binaries.

With a half run of 80 days, we are able to detect a significant fraction of the candidates found in the whole set of data. But it has to be understood that by shortening a run we lose information: we lose precision in the determination of the ephemeris, so the follow-up is more difficult; we lose really long period candidates; we lose precision in the transit timing variation analysis, because those need the longest time base line available; the oversampled (32 s) fraction of the light curve for planet candidates is shorter, so the characterization of their physical parameters is not as precise as with a longer baseline, etc. 


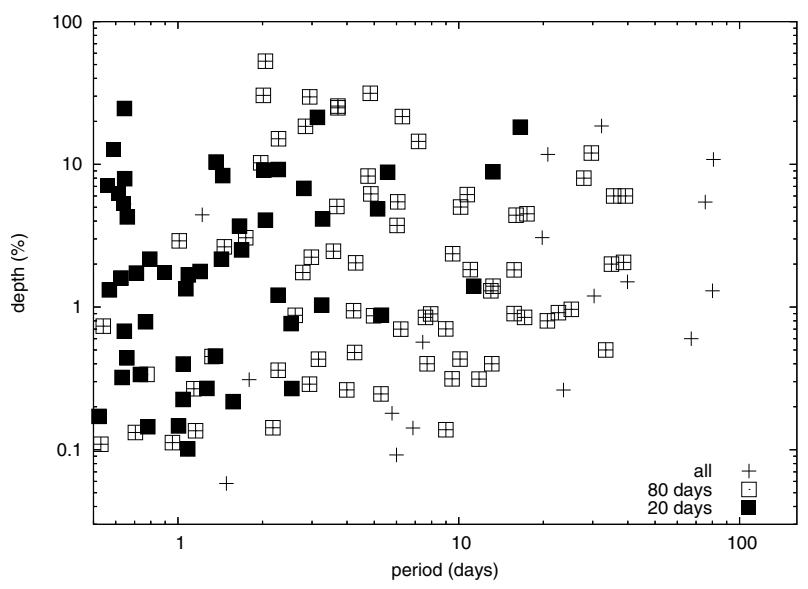

Fig. 15. Depths and periods of the candidates found in shortened runs of 20 and 80 days compared to the candidates found in the whole run.

Figure 15 shows a comparison of the depths and periods of the candidates found in different shortened runs. There are slight differences with Fig. 13 due to crowding effects and because the results presented in the comparative study are based only on the automatic pipeline of just one of the algorithms used by the detection teams. This diagram must not be used to make assertions on the detectability of a particular candidate, but as a tool for understanding the evolution of the efficiency of detection as a function of the length of the run (or, for a given period, the number of transits observed).

\section{Summary}

Table 3 summarizes the results of the follow-up effort done in the LRc01: 34 candidates with $R$ magnitudes between 12 and 16 were followed up with different photometric and spectroscopic facilities. The nature of 26 candidates has been unambiguously established: 2 planets, 7 contaminating eclipsing binaries, 3 blends, $6 \mathrm{SB} 1$, and $2 \mathrm{SB} 2$. Six binaries were rejected by CoRoT photometry. Finally, 16 candidates are waiting for follow-up. As already pointed out by Moutou et al. (2009), the follow-up requires a huge effort and it is not always straightforward or conclusive at the faint end of the magnitude range.

Figures 12 and 13 allow the question to be addressed of the detection capabilities of CoRoT. The signal of planets as small as Neptune should be detected in all the observed magnitude range. However, so far CoRoT has only found relatively large planets (with the exception of CoRoT-7b) at the bright end of the observed sample. A priori, nothing in the analyzed data indicates a severe degradation of the photometric performances beyond the expected photon noise limitations. The lack of Neptune and Jupiter-like planets at the faint end might be for two main reasons: first, the spectroscopic follow-up of the candidates is more complicated, requires longer observations and is in the reach of fewer instruments (namely HARPS). Second, some binaries can be easily identified as such from CoRoT photometry due to the presence of a secondary. However, it is more difficult to find this secondary around faint targets because the signal-to-noise ratio is poorer, which possibly makes the contamination of binaries higher at the faint end, reducing the performance of the planet detection. Recently, Almenara et al. (2009) have published a detailed study evaluating the rates and nature of false positives in the first CoRoT fields observed, which also provides insight into the nature of the planetary populations detected with CoRoT.
However, it cannot be discarded yet, as pointed out by Moutou et al. (2009), that there is an error found not in the detection of candidates, but in their selection: hence, good planetary candidates would have been rejected as binaries just from the analysis of photometric data because of a bad estimation of the planet parameters caused by poor knowledge of a faint host star. The possibility of a real lack of Jupiters around faint stars does not seem to have any physical basis.

For the 2 Earth radii limit, the constraints imposed by the photon noise limit are severe, and in the case of the LRc01, there is a lack of dwarf stars in the observed sample, which reduces the population of suitable targets for the planet search, hence the number of possible positive detections.

Acknowledgements. H.J.D., J.M.A., and M.R. acknowledge support by grants ESP2004-03855-C03-03 and ESP2007-65480-C02-02 of the Spanish Ministerio de Ciencia e Innovación. D.G. acknowledges the support of grant 50OW0204 from the Deutschen Zentrums für Luft- und Raumfahrt (DLR). T.M. acknowledges support from the Israel Science Foundation (grant No. 655/07).

Some of the data published in this article were acquired with the IAC 80 telescope operated by the Instituto de Astrofísica de Canarias in the Observatorio del Teide, and special thanks is given to its staff for performing a large fraction of these observations. Part of the observations presented in this paper were performed with the $2.1 \mathrm{~m}$ Otto Struve telescope operated at McDonald Observatory, and with the $2 \mathrm{~m}$ Alfred Jensch telescope at the Thüringer Landessternwarte Tautenburg. This research has made use of the Exo-Dat database, operated at LAM-OAMP, Marseille, France, on behalf of the CoRoT/Exoplanet program, whose input catalog was made possible thanks to observations collected for years at the Isaac Newton Telescope (INT), operated on the island of La Palma by the Isaac Newton group in the Spanish Observatorio del Roque de Los Muchachos of the Instituto de Astrofísica de Canarias.

This publication made use of NASA's Astrophysics Data System Bibliographic Services and of data products from the Two Micron All Sky Survey, which is a joint project of the University of Massachusetts and the Infrared Processing and Analysis Center/California Institute of Technology, funded by the National Aeronautics and Space Administration and the National Science Foundation.

\section{References}

Aigrain, S., Collier Cameron, A., Ollivier, M., et al. 2008, A\&A, 488, L43 Aigrain, S., Pont, F., Fressin, F., et al. 2009, A\&A, 506, 425

Alapini, A., \& Aigrain, S. 2008, in IAU Symp. 249, ed. Y.-S. Sun, S. FerrazMello, \& J.-L. Zhou, 89

Almenara, J. M., Deeg, H. J., Aigrain, S., et al. 2009, A\&A, 506, 337

Alonso, R., Auvergne, M., Baglin, A., et al. 2008, A\&A, 482, L21

Auvergne, M., Bodin, P., Boisnard, L., et al. 2009, A\&A, 506, 411

Baglin, A., Auvergne, M., Boisnard, L., et al. 2006, in COSPAR, Plenary Meeting, 36, 36th COSPAR Scientific Assembly, 3749

Barge, P. 2009, in IAU Symp., 253, 83

Barge, P., Baglin, A., Auvergne, M., et al. 2008a, A\&A, 482, L17

Barge, P., Baglin, A., Auvergne, M., \& the CoRoT team. 2008b, in IAU Symp., 249, 3

Baudin, F., Baglin, A., Orcesi, J.-L., et al. 2006, in ESA SP-1306, ed. M. Fridlund, A. Baglin, J. Lochard, \& L. Conroy, 145

Boisnard, L., \& Auvergne, M. 2006, in ESA SP-1306, ed. M. Fridlund, A. Baglin, J. Lochard, \& L. Conroy, 19

Bordé, P., Fressin, F., Ollivier, M., Léger, A., \& Rouan, D. 2007, in Transiting Extrapolar Planets Workshop, ed. C. Afonso, D. Weldrake, \& T. Henning, ASP Conf. Ser., 366, 145

Bouchy, F., Queloz, D., Deleuil, M., et al. 2008, A\&A, 482, L25

Burgasser, A. J., Dhital, S., \& West, A. 2009, AJ, accepted [arXiv: 0909. 3885]

Burrows, A., Hubbard, W. B., Lunine, J. I., \& Liebert, J. 2001, Rev. Mod. Phys., 73,719

Carpano, S., \& Fridlund, M. 2008, A\&A, 485, 607

Carpano, S., Cabrera, J., Alonso, R., et al. 2009, A\&A, 506, 491

Claret, A. 1998, A\&A, 335, 647

Claret, A. 2000, A\&A, 363, 1081

de Ridder, J., Barban, C., Baudin, F., et al. 2009, Nature, 459, 398

Debosscher, J., Sarro, L. M., López, M., et al. 2009, A\&A, 506, 519

Deeg, H. J., Gillon, M., Shporer, A., et al. 2009, A\&A, 506, 343

Defaÿ, C., Deleuil, M., \& Barge, P. 2001, A\&A, 365, 330 
Deleuil, M., Deeg, H. J., Alonso, R., et al. 2008, A\&A, 491, 889

Deleuil, M., Meunier, J. C., Moutou, C., et al. 2009, AJ, 138, 649

Drummond, R., Lapeyrere, V., Auvergne, M., et al. 2008, A\&A, 487, 1209

Gandolfi, D., Alcalá, J. M., Leccia, S., et al. 2008, ApJ, 687, 1303

Gondoin, P., et al. 2009, in Conf. proc. Cool Stars, 15

Hekker, S., Kallinger, T., Baudin, F., et al. 2009, A\&A, 506, 465

Karoff, C., Rauer, H., Erikson, A., et al. 2007, AJ, 134, 766

Lanza, A. F., Pagano, I., Leto, G., et al. 2009, A\&A, 493, 193

Léger, A., Rouan, D., Schneider, J., et al. 2009, A\&A, 506, 287

Llebaria, A., \& Guterman, P. 2006, in ESA SP-1306, ed. M. Fridlund, A. Baglin, J. Lochard, \& L. Conroy, 293

Mandel, K., \& Agol, E. 2002, ApJ, 580, L171

Mazeh, T., Guterman, P., Aigrain, S., et al. 2009, A\&A, 506, 431

Meunier, J.-C., Deleuil, M., Moutou, C., et al. 2007, in Astronomical Data Analysis Software and Systems XVI, ed. R. A. Shaw, F. Hill, \& D. J. Bell, ASP Conf. Ser., 376, 339

Michel, E., Baglin, A., Weiss, W. W., et al. 2008, Commun. Asteroseismol., 157, 69

Moutou, C., Pont, F., Barge, P., et al. 2005, A\&A, 437, 355
Moutou, C., Aigrain, S., Almenara, J., et al. 2007, in Transiting Extrapolar Planets Workshop, ed. C. Afonso, D. Weldrake, \& T. Henning, ASP Conf. Ser., 366, 127

Moutou, C., Bruntt, H., Guillot, T., et al. 2008, A\&A, 488, L47

Moutou, C., Pont, F., Bouchy, F., et al. 2009, A\&A, 506, 321

Pinheiro da Silva, L., Rolland, G., Lapeyrere, V., \& Auvergne, M. 2008, MNRAS, 384, 1337

Pont, F., Bouchy, F., Melo, C., et al. 2005, A\&A, 438, 1123

Pont, F., Zucker, S., \& Queloz, D. 2006, MNRAS, 373, 231

Press, W. H., Teukolsky, S. A., Vetterling, W. T., \& Flannery, B. P. 2002, Numerical Recipes in $\mathrm{C}++, 2$ nd edn. (Cambridge University Press)

Rauer, H., Queloz, D., Csizmadia, Sz., et al. 2009, A\&A, 506, 281

Régulo, C., Almenara, J. M., Alonso, R., Deeg, H., \& Roca Cortés, T. 2007, A\&A, 467, 1345

Renner, S., Rauer, H., Erikson, A., et al. 2008, A\&A, 492, 617

Seager, S., \& Mallén-Ornelas, G. 2003, ApJ, 585, 1038

Surace, C., Alonso, R., Barge, P., et al. 2008, in SPIE Conf., 7019

Torres, G., Winn, J. N., \& Holman, M. J. 2008, ApJ, 677, 1324

Wilson, R. E., \& Devinney, E. J. 1971, ApJ, 166, 605 
Table 3. Follow-up instruments used to solve the nature of the candidates in the LRc01.

\begin{tabular}{|c|c|c|c|c|}
\hline CoRoTid & Winid & $\bar{R}$ & Nature of the candidate & Follow-up facilities \\
\hline 101368192 & E1_0523 & 13.099 & CoRoT-3b & OGS, SOPHIE, Wise, BEST, IAC80, HARPS, TLS, McDonald \\
\hline 101206560 & E2_0192 & 12.204 & CoRoT-2b & IAC, Wise, SOPHIE, HARPS \\
\hline 100567226 & E2_5365 & 15.519 & SB1 & HARPS, FLAMES \\
\hline 110604224 & E2_3681 & 15.261 & CEB & EULER, IAC80 \\
\hline 101351899 & E2_0305 & 12.813 & SB1 & SOPHIE \\
\hline 101436549 & E1_3129 & 14.887 & CEB & IAC80, TLS \\
\hline 101068850 & E1_0769 & 13.511 & SB2 & SOPHIE \\
\hline 100589010 & E2_4006 & 15.379 & CEB & HARPS, Wise \\
\hline 100609705 & E2_3895 & 15.313 & CEB & EULER, IAC80 \\
\hline 101653417 & E1_1253 & 13.988 & blend & HARPS, SOPHIE \\
\hline 100982006 & E1_1090 & 13.643 & SB1 & SOPHIE \\
\hline 101708400 & E1_2936 & 14.788 & SB1 & FLAMES \\
\hline 101482707 & E1_2837 & 12.969 & SB2 & SOPHIE \\
\hline 101031117 & E2_0840 & 13.644 & SB1 & SOPHIE \\
\hline 100773735 & E2_1245 & 13.740 & SB1(?) & SOPHIE \\
\hline 101259269 & E1_5626 & 15.421 & binary & rejected from CoRoT photometry \\
\hline 101218359 & E1_1845 & 14.612 & binary & rejected from CoRoT photometry \\
\hline 100468104 & E2_2533 & 14.733 & binary & rejected from CoRoT photometry \\
\hline 100834293 & E2_5414 & 15.948 & binary & IAC80, rejected from CoRoT photometry \\
\hline 101026464 & E1_5745 & 15.937 & binary & rejected from CoRoT photometry \\
\hline 101434308 & E1_3425 & 14.477 & binary & rejected from CoRoT photometry \\
\hline 101106246 & E2_3257 & 15.345 & CEB & EULER, IAC80 \\
\hline 101095286 & E1_2376 & 11.907 & CEB & Wise, IAC 80 \\
\hline 101175376 & E1_4959 & 15.377 & CEB & IAC 80 \\
\hline 101055792 & E1_2140 & 14.570 & blend & IAC80, SOPHIE, HARPS, TLS \\
\hline 101675703 & E1_4863 & 14.765 & blend & HARPS \\
\hline 101086161 & E2_1145 & 14.267 & unknown & SOPHIE, OHP, HARPS \\
\hline 100905839 & E2_5801 & 15.673 & unknown & HARPS, UVES \\
\hline 100725706 & E2_1802 & 13.744 & unknown & HARPS \\
\hline 101206989 & E1_1929 & 14.449 & unknown & IAC80, SOPHIE, HARPS, TLS \\
\hline 101332685 & E2_3345 & 15.250 & unknown & HARPS \\
\hline 101186644 & E1_4780 & 15.670 & unknown & HARPS \\
\hline 101157411 & E1_0715 & 13.308 & unknown & SOPHIE, UVES \\
\hline 101123916 & E2_4295 & 15.868 & unknown & OGS, CFHT \\
\hline 101424939 & E1_1632 & 14.490 & unknown & EULER \\
\hline 100768215 & E2_4390 & 15.875 & unknown & IAC 80, CFHT \\
\hline 100887662 & E2_0934 & 13.812 & unknown & not started \\
\hline 100980128 & E1_0673 & 13.155 & unknown & TLS \\
\hline 101439653 & E1_2761 & 14.758 & unknown & not started \\
\hline 101115531 & E1_1791 & 14.436 & unknown & not started \\
\hline 100654833 & E2_4241 & 14.577 & unknown & FLAMES \\
\hline 101614469 & E1_2278 & 13.880 & unknown & HARPS, TLS \\
\hline
\end{tabular}

Table 4. Parameters of the candidates in ongoing follow-up.

\begin{tabular}{|c|c|c|c|c|c|c|c|c|c|c|c|c|}
\hline $\mathrm{pr}$ & CoRoTid & $\overline{\text { Winid }}$ & $\begin{array}{c}\text { Right ascension } \\
(\mathrm{J} 2000.0)\end{array}$ & $\begin{array}{c}\text { Declination } \\
(\mathrm{J} 2000.0)\end{array}$ & $\bar{B}$ & $\bar{V}$ & $\bar{R}$ & $\bar{I}$ & $\overline{\text { Period(d) }}$ & $\begin{array}{c}\text { Epoch } \\
\text { (HJD-2 } 454 \text { 000) }\end{array}$ & $\begin{array}{r}\text { Depth } \\
(\%)\end{array}$ & $\begin{array}{l}\text { ngth } \\
\text { (h) }\end{array}$ \\
\hline 1 & 101086161 & E2_1145 & 192621.25 & 42535.3 & 16.096 & 14.797 & 14.267 & 13.408 & $42+0.00$ & $282.5214 \pm 0.0007$ & 0.69 & 2.3 \\
\hline 2 & & 1 & & & & & & & & 25 & & .9 \\
\hline 2 & 06 & 2 & & & & & & & & & & .2 \\
\hline 4 & 89 & 1_1929 & 1 & & 8 & 2 & 49 & 13 & 0. & 2 & 30 & 3 \\
\hline 3 & 85 & 23345 & 1 & & 5 & 77 & 15 & 6 & 13.09 & 0 & 0.48 & 3.6 \\
\hline 2 & 644 & 1_4780 & 1 & & 2 & 49 & 70 & 4 & 40.3 & 7 & 0.86 & 5.9 \\
\hline 3 & 411 & E1_0715 & 192 & -00649.8 & .426 & 48 & .308 & 12.822 & & 28 & 0.60 & 9. \\
\hline 3 & 23916 & 24295 & 1926 & 6 & 872 & 49 & 15.868 & 15.048 & 00050 & 50 & 0.28 & 2.9 \\
\hline 3 & +24939 & 1 1632 & 28 & 2.5 & 864 & 356 & .490 & 35 & 0033 & 14 & 4 & 1.2 \\
\hline 3 & 215 & 4390 & 1 & 6.3 & 52 & 64 & 75 & 2 & 019 & 4 & 0 & 4.1 \\
\hline 4 & 662 & 2 & 1925 & 7.5 & 8 & 8 & 12 & 47 & & 50 & 28 & 8.6 \\
\hline 4 & 28 & 73 & & & & & & 39 & & & 0.09 & 2.9 \\
\hline 3 & & 61 & & -0 & & & & & & & 4.11 & 3.1 \\
\hline 4 & & 1_1791 & & & & & & & 0.5 & & 0.18 & 0.96 \\
\hline 3 & & 2_4241 & & & & & & & & & 0. & 2.3 \\
\hline 3 & 101614469 & E1_2278 & 192956.79 & 00553.3 & 16.591 & 15.231 & 14.673 & 13.880 & $6.86934 \pm 0.00067$ & $242.0216 \pm 0.0095$ & 0.16 & 0.1 \\
\hline
\end{tabular}


Table 5. Parameters of the candidates solved by the follow-up. The values for the period and epoch for CoRoT-2b and CoRoT-3b have been taken from the literature.

\begin{tabular}{|c|c|c|c|c|c|c|c|c|c|c|c|c|c|}
\hline$\overline{\mathrm{pr}}$ & CoRoTid & Winid & $\begin{array}{c}\text { Right ascension } \\
(\mathrm{J} 2000.0)\end{array}$ & $\begin{array}{c}\text { Declination } \\
(\mathrm{J} 2000.0)\end{array}$ & $\overline{\bar{B}}$ & $\overline{\bar{V}}$ & $\overline{\bar{R}}$ & $\overline{\bar{I}}$ & $\overline{\overline{P e r i o d}(\mathrm{~d})}$ & $\begin{array}{c}\text { Epoch } \\
\text { (HJD-2 } 454 \text { 000) }\end{array}$ & $\begin{array}{r}\text { Depth } \\
(\%)\end{array}$ & $\begin{array}{r}\begin{array}{l}\text { Length } \\
\text { (h) }\end{array}\end{array}$ & $\begin{array}{l}\text { Follow-up } \\
\text { result }\end{array}$ \\
\hline 1 & 101368192 & E1_0523 & 192813.26 & 00718.7 & 14.199 & 13.292 & 13.099 & 12.676 & $4.25680 \pm 0.000005$ & $283.1383 \pm 0.0003$ & 0.45 & 3.5 & CoRoT-3b \\
\hline 1 & 101206560 & E2_0192 & 192706.50 & 12301.5 & 13.422 & 12.568 & 12.204 & 11.641 & $1.7429964 \pm 0.0000017$ & $237.5356 \pm 0.0007$ & 2.9 & 1.9 & CoRoT-2b \\
\hline 1 & 100567226 & E2_5365 & 192315.71 & 11845.2 & 17.089 & 15.974 & 15.519 & 14.861 & $7.70975 \pm 0.00001$ & $244.3781 \pm 0.0001$ & 0.47 & 1.8 & SB1 \\
\hline 1 & 110604224 & E2_3681 & 192402.74 & 04907.6 & 16.752 & 15.684 & 15.261 & 14.471 & $3.45075 \pm 0.00001$ & $240.5668 \pm 0.0001$ & 0.15 & 2.8 & CEB \\
\hline 2 & 101351899 & E2_0305 & 192805.19 & 12223.7 & 13.819 & 13.079 & 12.813 & 12.330 & $21.97190 \pm 0.00069$ & $239.0418 \pm 0.0021$ & 0.93 & 3.6 & SB1 \\
\hline 3 & 101436549 & E1_3129 & 192842.75 & 03345.0 & 15.984 & 15.204 & 14.887 & 14.750 & $0.73495 \pm 0.00050$ & $237.2723 \pm 0.0050$ & 0.30 & 1.4 & CEB \\
\hline 4 & 101068850 & E1_0769 & 192613.35 & 03935.2 & 15.194 & 14.007 & 13.511 & 12.632 & single transit & $294.4770 \pm 0.0050$ & 4.0 & 7.2 & SB2 \\
\hline 3 & 100589010 & E2_4006 & 193123.52 & 11622.7 & 17.243 & 15.934 & 15.379 & 14.591 & $0.78053 \pm 0.00050$ & $241.0131 \pm 0.0050$ & 0.14 & 2.0 & CEB \\
\hline 2 & 100609705 & E2_3895 & 193130.84 & 11432.6 & 16.968 & 15.813 & 15.313 & 14.685 & $3.30382 \pm 0.00001$ & $242.8553 \pm 0.0001$ & 0.09 & 4.0 & CEB \\
\hline 3 & 101653417 & E1_1253 & 193017.21 & 00932.9 & 16.027 & 14.591 & 13.988 & 13.215 & $5.77991 \pm 0.00050$ & $239.0721 \pm 0.0050$ & 0.15 & 2.5 & blend \\
\hline 3 & 100982006 & E1_1090 & 192537.52 & 01031.0 & 15.039 & 14.052 & 13.643 & 13.092 & $16.68904 \pm 0.00050$ & $236.9460 \pm 0.0050$ & 0.40 & 3.8 & SB1 \\
\hline 3 & 101708400 & E1_2936 & 193040.09 & -00108.9 & 16.436 & 15.256 & 14.788 & 14.165 & $4.220221 \pm 0.000015$ & $279.2119 \pm 0.0009$ & 0.85 & 5.1 & SB1 \\
\hline 2 & 101482707 & E1_2837 & 192901.57 & 03712.2 & 14.474 & 13.366 & 12.969 & 12.256 & $39.8883 \pm 0.0001$ & $260.2825 \pm 0.00013$ & 1.31 & 4.9 & SB2 \\
\hline 3 & 1117 & E2_0840 & 192556.12 & 13200.5 & 14.767 & 13.937 & 13.644 & 13.008 & $107.5878=$ & $313.4829 \pm 0.0048$ & 0.60 & 3.8 & SB1 \\
\hline 2 & 100773735 & E2_1245 & 192431.35 & 11315.9 & 14.837 & 14.056 & 13.740 & 13.232 & $4.973876 \pm 0.000008$ & $279.5044 \pm 0.0004$ & 0.66 & 4.4 & SB2 \\
\hline 4 & 101259269 & E1_5626 & 192726.48 & 04503.5 & 17.414 & 15.942 & 15.421 & 14.437 & $4.43324 \pm 0.00025$ & $240.9053 \pm 0.0055$ & 0.21 & 4.8 & binary \\
\hline 4 & 101218359 & E1_1845 & 192710.96 & -02750.3 & 16.599 & 15.196 & 14.612 & 13.855 & $1.154871 \pm 0.000024$ & $237.6010 \pm 0.0015$ & 0.13 & 1.0 & binary \\
\hline 4 & 100468104 & E2_2533 & 192240.22 & 13406.8 & 16.390 & 15.228 & 14.733 & 14.032 & $1.043594 \pm 0.000009$ & $237.0941 \pm 0.0007$ & 0.32 & 1.6 & binary \\
\hline 4 & 100834293 & E2_5414 & 192450.46 & 14511.4 & 17.308 & 16.358 & 15.948 & 15.268 & $11.301870 \pm 0.000024$ & $275.8783 \pm 0.0013$ & 0.90 & 2.3 & binary \\
\hline 4 & 101026464 & E1_5745 & 192554.05 & -01724.5 & 17.174 & 16.311 & 15.937 & 15.425 & $1.322011 \pm 0.000019$ & $238.1208 \pm 0.0012$ & 0.40 & 1.1 & binary \\
\hline 4 & 101434308 & E1_3425 & 192841.80 & -02409.5 & 15.659 & 14.846 & 14.477 & 14.035 & $79.9808 \pm 0.0005$ & $280.6340 \pm 0.0050$ & 2.7 & 10.0 & binary \\
\hline 2 & 101106246 & E2_3257 & 192628.79 & 10045.6 & 17.639 & 15.983 & 15.345 & 14.389 & $11.31662 \pm 0.00007$ & $252.7348 \pm 0.0043$ & 0.33 & 3.6 & CEB \\
\hline 4 & 101095286 & E1_2376 & 192624.66 & 03606.1 & 15.294 & 13.552 & 12.986 & 11.907 & $10.10655 \pm 0.00050$ & $241.2432 \pm 0.0050$ & 0.25 & 4.2 & CEB \\
\hline 4 & 101175376 & E1_4959 & 192654.86 & -01056.9 & 16.554 & 15.731 & 15.377 & 14.850 & $5.28013 \pm 0.00031$ & $239.0282 \pm 0.0005$ & 0.41 & 11.9 & CEB \\
\hline 1 & 101055792 & E1_2140 & 192607.32 & 01631.6 & 16.400 & 15.112 & 14.570 & 13.836 & $1.9384 \pm 0.0001$ & $237.6745 \pm 0.0001$ & 0.13 & 2.0 & blend \\
\hline 1 & 101675703 & E1_4863 & 193026.34 & 00109.9 & 17.424 & 16.091 & 15.487 & 14.765 & $1.79308 \pm 0.00050$ & $238.0019 \pm 0.0050$ & 0.29 & 2.4 & blend \\
\hline
\end{tabular}

Table 6. Eclipsing binaries found in LRc01.

\begin{tabular}{|c|c|c|c|c|c|c|c|c|c|c|c|}
\hline CoRoTid & Winid & $\begin{array}{c}\text { Right ascension } \\
(\mathrm{J} 2000.0)\end{array}$ & $\begin{array}{c}\text { Declination } \\
(\mathrm{J} 2000.0)\end{array}$ & $\bar{B}$ & $\bar{V}$ & $\bar{R}$ & $\bar{I}$ & $\overline{P e r i o d}(\mathrm{~d})$ & $\begin{array}{c}\text { Epoch } \\
\text { (HJD-2 454 000) }\end{array}$ & $\begin{array}{r}\text { Length } \\
\text { (h) }\end{array}$ & $\begin{array}{r}\text { Depth } \\
(\%)\end{array}$ \\
\hline 100493215 & $2 \_3956$ & 192249.06 & 12315.7 & 686 & .531 & .043 & .345 & $0.14815 \pm 0.000179$ & $40.603937 \pm 0.001461$ & 9.1 & 3.5 \\
\hline 24672 & 2_4093 & & & & & & & 00006 & & 2.74 & 11.0 \\
\hline 34603 & 2_1954 & 2 & 8.1 & 512 & 955 & 326 & 92 & 00232 & 379 & 8.3 & 1.4 \\
\hline 100542479 & 2_4680 & 192306.71 & 2009.6 & 16.916 & .911 & 5.480 & .858 & single eclipse & $263.886 \pm 0$ & 8.4 & 4.0 \\
\hline 100552362 & 2_4218 & 192310.26 & 2237.6 & 16.453 & .588 & .236 & 4.705 & $347553 \pm 0.000$ & $7.988331 \pm$ & 12.0 & 1.9 \\
\hline 7689 & 2_5511 & 87 & 225.7 & .150 & .051 & 573 & 938 & & & 7.0 & 4.4 \\
\hline 369 & 2_3526 & 0 & 5.4 & & & & & & & 1.35 & 3.2 \\
\hline 7133 & $2 \_4820$ & 19232 & 9947.3 & 17.534 & 16.314 & .814 & 101 & .000045 & $239.260664 \pm$ & 6.4 & 0.9 \\
\hline 58 & 2_2864 & 1923 & 239.4 & 16.666 & 528 & 13 & 58 & 00008 & 30 & 2.6 & 0.4 \\
\hline & 2_4283 & & & & & & & & & 2.3 & 2 \\
\hline 853 & 2_5219 & 192 & 4.3 & 107 & 64 & & & & & 1.71 & 0.2 \\
\hline 622251 & 2_2631 & 1923 & 1058.7 & 954 & 531 & & 052 & 2166 & 262.3 & .65 & 0.7 \\
\hline 08 & 2_5789 & 4 & & & & & & & 3 & 37 & 8.8 \\
\hline & 2_3828 & & & & & & & & & .00 & 2.7 \\
\hline 547 & 2_5020 & 192 & 3.8 & & 83 & 77 & & 02 & & 3.54 & 5.6 \\
\hline 80 & 2_1661 & 99 & & & & & & & & 8.4 & 20.7 \\
\hline & 2_4423 & & & & & & & & & 2. & \\
\hline 60 & _1868 & 9 & & & & & & & & 1.0 & 0.5 \\
\hline 721 & 2_5407 & 12 & & 7.078 & & 723 & 015 & & & 7.3 & 20.8 \\
\hline 31 & 2_5524 & 2 & & & & & & 05 & 97 & 3.03 & 1. \\
\hline & 2_0667 & 2 & & & & & & & & 2.9 & \\
\hline 14 & __3570 & 192 & & 98 & 13 & 43 & 83 & & & .06 & 1.9 \\
\hline 622 & 2_5567 & 3 & & & & & & & & 9.3 & 12.8 \\
\hline & 2_2130 & 2 & & & & & & & 0 & 3.8 & 0.95 \\
\hline 11 & 2_0520 & 6 & .1 & 3 & 8 & 8 & 9 & & & 5.7 & 2.6 \\
\hline 3487 & 2_1078 & 1924 & & & & & & 00004 & 237.78 & 3.3 & 8.8 \\
\hline & 2_0379 & 192 & & & 12.521 & & 12.120 & & & 9.0 & 12.7 \\
\hline & $2-3910$ & 13 & & & & & & & $2+0.2$ & 9.7 & 6 \\
\hline & 225563 & 1024 & & 16.632 & & & & & & 3.8 & 6.0 \\
\hline 100773527 & $1 \_2325$ & 1924 & 02826.5 & 16.009 & 15.069 & 14.681 & 13.991 & .000034 & $238.792951 \pm 0$ & 3.28 & 0.1 \\
\hline & 2 _o & & & & & & & & & 7.2 & 6.2 \\
\hline 100794107 & 2 2_3582 & 19243 & & & & & & & 237.61 & 1.32 & \\
\hline 100805120 & $2 \_0271$ & 1924 & 03713.6 & 15.066 & 13.546 & 13.120 & & & 239.41 & 4.1 & 1.6 \\
\hline 100811279 & E2_2752 & 1924 & 11237.6 & 16.667 & 15.521 & 15.029 & 14.340 & $0.339217 \pm 0.000007$ & $237.652542 \pm 0.001572$ & 2.5 & 1.2 \\
\hline 100813794 & E2_0401 & 192444.02 & 14221.3 & 14.299 & 13.438 & 13.133 & 12.498 & $1.083353 \pm 0.000011$ & $237.610906 \pm 0.000859$ & 1.40 & 0.1 \\
\hline
\end{tabular}


Table 6. continued.

\begin{tabular}{|c|c|c|c|c|c|c|c|c|c|c|c|}
\hline CoRoTid & $\overline{\text { Winid }}$ & $\begin{array}{c}\text { Right ascension } \\
\text { (J2000.0) }\end{array}$ & $\begin{array}{c}\text { Declination } \\
(\mathrm{J} 2000.0)\end{array}$ & $\bar{B}$ & $\bar{V}$ & $\bar{R}$ & $\overline{I I}$ & $\overline{\text { Period(d) }}$ & $\begin{array}{c}\text { Epoch } \\
\text { (HJD-2 454 000) }\end{array}$ & $\begin{array}{r}\begin{array}{l}\text { Length } \\
\text { (h) }\end{array} \\
\end{array}$ & $\begin{array}{r}\text { Depth } \\
(\%) \\
\end{array}$ \\
\hline 100818164 & E1_1319 & 192445.40 & 02731.8 & 15.419 & 14.409 & .066 & 3.340 & $306873 \pm 0.000001$ & $37.275349 \pm 0.000172$ & 2.7 & 3.8 \\
\hline 854 & E2_2257 & & & & & & & & & 2.31 & \\
\hline 100851348 & 1 & 192455.87 & 01945.0 & 15.867 & 14.913 & 14.591 & 13.976 & $4829 \pm 0.000004$ & $237.713793 \pm 0.000440$ & 8.7 & 1.6 \\
\hline 100866999 & E2_1719 & 192500.78 & 05441.1 & 15.867 & 14.971 & 14.599 & 13.980 & $08769 \pm 0.000022$ & $239.396628 \pm 0.000656$ & 3.9 & 6.7 \\
\hline & & & & & & & & & & 2.7 & $\rho_{-1}$ \\
\hline 1803 & E1_3671 & 192502.31 & 01852.9 & 16.563 & 15.502 & 15.086 & 14.447 & $7.439679 \pm 0.000202$ & $243.026515 \pm 0.002314$ & 2.59 & 0.4 \\
\hline 100878452 & E2_4445 & 192504.40 & 10541.0 & 17.972 & 16.486 & 15.871 & 14.972 & $1.359027 \pm 0.000018$ & $238.398893 \pm 0.001068$ & 2.29 & 0.4 \\
\hline & 20431 & 192 & 50.7 & 15.123 & 14.588 & 4.413 & 13.964 & & 237. & 9.4 & 1.9 \\
\hline 100883990 & $2 \_4314$ & 192506.18 & 15051.1 & 16.968 & 15.871 & 15.433 & 15.205 & $0.848685 \pm 0.000008$ & $237.619000 \pm 0.000751$ & 1.10 & 0.2 \\
\hline 5002 & 4653 & 192506.49 & 15031.3 & 15.938 & 14.610 & 14.086 & 13.327 & $11.808625 \pm$ & $248.643047 \pm$ & 5.04 & 0.5 \\
\hline 3207 & 3579 & 192 & 33.8 & 16.164 & 15.453 & 15.196 & 14.640 & $6.030804 \pm 0$. & $9856 \pm 0$ & 5.85 & 3.1 \\
\hline 100906796 & 20663 & 192513.40 & 15710.6 & 14.172 & 13.597 & 13.427 & 13.075 & $0.897635 \pm 0.000006$ & $237.431242 \pm 0.000504$ & 8.6 & 1.5 \\
\hline 9405 & 0377 & 80 & & 15.069 & & 463 & 972 & & & & 3.3 \\
\hline 100921311 & 0777 & 192518.09 & 02912.6 & 15.609 & 13.954 & 13.330 & 12.371 & .000017 & & 3.9 & 0.76 \\
\hline 100934790 & 4048 & 192 & 01738.3 & 17.588 & 16.437 & 15.966 & 15.282 & $0.276416 \pm 0.000005$ & $9950 \pm 0$ & 0.60 & 0.6 \\
\hline 095 & 3518 & & & 17.152 & 15.616 & .970 & 107 & & 276 & 47 & $5=$ \\
\hline 100950734 & 5104 & 192 & 04423.0 & 17.156 & 15.906 & 15.550 & 4.644 & .000002 & $8086 \pm 0$ & 3.54 & 0.9 \\
\hline 3598 & 24803 & 192 & 00.8 & 17.245 & 16.058 & 15.603 & 14.798 & & 238 & 1.81 & 1.9 \\
\hline & 0545 & & & 14.785 & 771 & 476 & 748 & & & 2.0 & 4.4 \\
\hline 100964052 & 4946 & 192 & 05740.6 & 17.851 & 16.406 & 15.749 & 14.938 & $0718631+$ & $1966+0$ & 2.21 & 0.3 \\
\hline 4422 & 2907 & 19 & 20.9 & 16.305 & 15.394 & 15.013 & 4.337 & & & 3.0 & 3.7 \\
\hline & 0747 & & & 15.497 & 15.019 & & & & & .5 & 16.0 \\
\hline 100972787 & 3478 & & 7.2 & 16.443 & 15.541 & 15.163 & 569 & 05 & 75 & 3.54 & 0.4 \\
\hline 100998053 & 2588 & 192 & 7.1 & 16.121 & 15.314 & 4.944 & 68 & & & 4.0 & 27.4 \\
\hline 813 & 5271 & & -0 & 17.270 & 16 & 763 & 26 & & & 8.2 & 25.3 \\
\hline 101006984 & 1872 & & & 15.985 & 15.050 & 14.619 & & & 23 & 5.56 & 0.2 \\
\hline 567 & 3938 & 02 & 35.3 & 16.626 & 15.850 & 15.559 & 4.899 & & 23 & 5.2 & 5.1 \\
\hline 476 & 4075 & & -00 & 16.351 & 15.522 & 15.160 & 14.627 & & 2. & 1.43 & 0.3 \\
\hline & 2433 & & $-01^{1}$ & 16.314 & 15.201 & 14.717 & 14.065 & & & 1.8 & 1.8 \\
\hline & -0249 & & & 15.227 & 14.504 & & & & & 4.4 & 6.6 \\
\hline 101017242 & 297 & & & & & & & 11 & & 4.2 & .9 \\
\hline & _2978 & & 8.4 & 16.014 & 15.171 & & & & & 6.2 & 12.9 \\
\hline & 1450 & & & 16.127 & & & & & & .68 & 14.1 \\
\hline 21491 & E2_2192 & & & & & & & & & 0 & 8.0 \\
\hline & 3995 & & & 15.924 & 1 & 51 & 92 & 31 & & .0 & 0.4 \\
\hline 167 & 2861 & & & 16.886 & 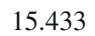 & & & & & 4.0 & 3.9 \\
\hline & 2871 & & & & & & & & & & .9 \\
\hline 194 & 0436 & & & 15.406 & 14.020 & 54 & 94 & & 2 & 2.5 & 21.0 \\
\hline 4418 & 3462 & & & 17.546 & 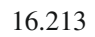 & 24 & 51 & & & .65 & 0.4 \\
\hline & +1 & & & & & & & & & & .9 \\
\hline & 10 & & & 17 & & & & & & 4 & 0.7 \\
\hline & 94 & & & 16 & & & 09 & & & 63 & 1.0 \\
\hline & 0400 & & & & & & & & & 3.2 & .2 \\
\hline & 5665 & & & & & & 3.001 & & & 5.12 & 0.5 \\
\hline & 4987 & & & & & & & & & 2.9 & 3.6 \\
\hline & 77 & & & & & & & & & 1.16 & 0.2 \\
\hline & 2851 & & & 16 & & & & & & 2.6 & 6.3 \\
\hline & & & & & & & & & & 5.7 & 0.7 \\
\hline & 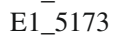 & & & & & & & & & 1 & - \\
\hline & 4013 & & & & & & & & & 1.9 & 3.7 \\
\hline & & & & & & & & & & 3.2 & 22.8 \\
\hline & & & & & & & & & & 52 & 26.7 \\
\hline 378 & 5245 & & & 17.339 & & & & & & 4.3 & 7.7 \\
\hline & & & & & & & & & & & \\
\hline & 0713 & & & & & & & & & 1.74 & 2 \\
\hline & & & & & & & & & & 49 & 12 \\
\hline & & & & & & & & & & 1 & \\
\hline & & & & & & & & & & 19 & \\
\hline & 226 & & & 12.790 & & & & & & 3 & 0.1 \\
\hline & & & & & & & & & & & \\
\hline & & & & & & & & & & 7.2 & 17.8 \\
\hline & 4273 & & & & & & & & & 4.07 & .6 \\
\hline & & & & & & & & & & & 09 \\
\hline 2008 & E2_4406 & & & 17. & 16. & & 14.597 & 22 & 241.0 & 8.7 & 24.5 \\
\hline & E2_2999 & & & & & & & & & .73 & 8 \\
\hline & 2365 & & & & & & & & & 3.80 & 1 \\
\hline 101183660 & E2_2553 & 192657.96 & 2103.8 & 15.642 & 14.504 & 14.024 & 13.248 & $9.464590 \pm 0.000731$ & $245.172271 \pm 0.005533$ & 5.69 & 0. \\
\hline
\end{tabular}


Table 6. continued.

\begin{tabular}{|c|c|c|c|c|c|c|c|c|c|c|c|}
\hline$\overline{\text { CoRoTid }}$ & $\overline{\text { Winid }}$ & $\begin{array}{c}\text { Right ascension } \\
(\mathrm{J} 2000.0)\end{array}$ & $\begin{array}{c}\text { Declination } \\
(\mathrm{J} 2000.0)\end{array}$ & $\bar{B}$ & $\overline{\bar{V}}$ & $\bar{R}$ & $\bar{I}$ & $\overline{\text { Period }(\mathrm{d})}$ & $\begin{array}{c}\text { Epoch } \\
\text { (HJD-2 454 000) }\end{array}$ & $\begin{array}{r}\text { Length } \\
\text { (h) }\end{array}$ & $\begin{array}{r}\text { Depth } \\
(\%) \\
\end{array}$ \\
\hline 418 & E2_3034 & 192700.48 & 15435.9 & 8 & 15.455 & 22 & 4 & 1 & $9.298109 \pm 0.000309$ & 7.2 & \\
\hline & 2459 & - & & & & & & 0001 & & & \\
\hline 550 & 3684 & & 51.1 & & .012 & & 2.372 & 0006 & & 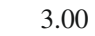 & \\
\hline 101205718 & 1352 & & 2921.5 & 57 & .729 & 205 & 3.274 & $339189 \pm 0.002361$ & $66.615760 \pm 0.006101$ & 6.76 & .1 \\
\hline 9902 & 4232 & & & & & & & & & 25 & \\
\hline 2999 & 5097 & 192708.94 & & 32 & .094 & & 5.034 & & & 6.8 & 4.8 \\
\hline 101213599 & I_2727 & 192709.15 & -00536.9 & 15.953 & 1.972 & 1.538 & 13.911 & $0.59042 \pm 0.00010$ & $236.98500 \pm 0.000500$ & 1.8 & 13.5 \\
\hline & & & & & & & & & & 2.6 & \\
\hline 1012 & 1891 & 19271 & 2.3 & 769 & .749 & & 560 & & & 6.0 & 4.3 \\
\hline 1012 & 4077 & 10 & 1705.3 & .884 & .011 & 1.627 & 4.045 & & & 4.54 & 0.3 \\
\hline & & & & & & & & & & & \\
\hline & & 192 & 9.4 & 33 & & & & & & 8.2 & 45.7 \\
\hline & 4910 & & & .674 & 208 & .613 & .676 & & & 47 & 0.6 \\
\hline & & & & & & & & & & 3.5 & \\
\hline & 3 & & & & & & & & & 16 & 0.5 \\
\hline & -2 & & & & & & & & & 0.66 & \\
\hline & & & & & & & & & & 8.2 &.$J$ \\
\hline & _0641 & & & 73 & & & & & & 6.0 & 0.3 \\
\hline & & & & & & & & & & 8.0 & \\
\hline & & & & & & & & & & .8 & .4 \\
\hline & $-5 c 0$ & & & & & & & & & 6 & 1 \\
\hline & & & & & & & & & & & \\
\hline & & & & & & & & & & 6.7 & .2 \\
\hline & & & & & & & & & & & \\
\hline & & & & & & & & & & & .0 \\
\hline & & & & & & & & & & & .7 \\
\hline & & & & & & & & & & .9 & \\
\hline & & & & & & & & & & 0.0 & .0 \\
\hline & & & & & & & & & & 4.5 & .35 \\
\hline & & & & & & & & & & & \\
\hline & & & & & & & & & & & .8 \\
\hline & & & & & & & & & & & \\
\hline & & & & & & & & & & 52 & \\
\hline & & & & & & & & & & 5.6 & 7.0 \\
\hline & & & & & & & & & & . & \\
\hline & & & & & & & & & & & 8.8 \\
\hline & & & & & & & & & & 01 & 2.0 \\
\hline & & & & & & & & & & & \\
\hline & & & & & & & & & & & 1 \\
\hline & & & & & & & & & & .0 & 3.5 \\
\hline & & & & & & & & & & & \\
\hline & & & & & & & & & & .0 & 3.7 \\
\hline & & & & & & & & & & & .4 \\
\hline & & & & & & & & & & & \\
\hline & 1 & & & & & & & & & 7.21 & 0.3 \\
\hline & & & & & & & & & & 3.0 & 1.0 \\
\hline & & & & & & & & & & & \\
\hline & -12 & & & & & & & & & 4.5 & 9.0 \\
\hline & & & & & & & & & & & 1.0 \\
\hline & & & & & & & & & & & \\
\hline & $L_{-}$ & & & & & & & & & 06 & 10.8 \\
\hline & & & & & & & & & & & \\
\hline & & & & & & & & & & & \\
\hline & & & & & & & & & & & \\
\hline & & & & & & & & & & & \\
\hline & & & & & & & 3.145 & \pm 0.000012 & $237.747480 \pm 0.001064$ & 1.40 & \\
\hline
\end{tabular}

